\title{
LA CORTE DI CASSAZIONE RITORNA SUL TEMA DEL RICONOSCIMENTO DEL RIPUDIO ISLAMICO
}

\author{
THE ITALIAN CORTE DI CASSAZIONE RETURNS ON \\ THE ISSUE OF RECOGNITION OF ISLAMIC REPUDIATIONS
}

\author{
Francesco Pesce \\ Professore associato di Diritto internazionale \\ Università degli Studi di Genova, Italy
}

Recibido: 08.01.2021 / Aceptado: 14.01.2021

DOI: https://doi.org/10.20318/cdt.2021.5970

\begin{abstract}
Riassunto: Con sentenza depositata il 7 agosto 2020, la Corte di cassazione è tornata a confrontarsi con il tema del riconoscimento di un provvedimento straniero di ripudio. Come noto l'istituto, proprio degli ordinamenti giuridici di matrice islamica, è già stato oggetto in passato di particolari attenzioni da parte della giurisprudenza italiana, prevalentemente orientata nel senso di negare l'attribuzione di qualsiasi effetto a causa di un insormontabile conflitto "ontologico" tra i valori sottesi a tale istituto nei Paesi d'origine ed i principi fondanti il cd. ordine pubblico internazionale del foro interno. Il contributo mira a vagliare in senso critico tale posizione, anche alle luce di alcuni isolati segnali di segno opposto, rinvenibili sia in giurisprudenza che nella riflessione dottrinale.

Parole chiave: ripudio, riconoscimento di decisioni straniere, ordine pubblico, famiglia, tutela dei diritti fondamentali.

Abstract: With its judgment filed on 7 August 2020, the Italian Corte di cassazione addressed, once again, the issue of the recognition of a foreign repudiation order. As it is well known, the institution of repudiation, which is traditional within Islamic legal systems, has already been the subject of a specific attention in the past in the Italian case-law, which was mainly oriented towards denying the attribution of any effect because of an insurmountable "ontological" conflict between the values underlying this institution in the countries of origin with the principles underlying the so-called international public order of the domestic forum. The contribution aims to examine this position critically, also moving from some isolated signs of a different view, to be found both in the case-law and in the related literature.
\end{abstract}

Keywords: repudiation, recognition of foreign decisions, public policy, family, protection of human rights.

Sommario: I. La Cassazione ritorna sul riconoscimento di un ripudio islamico. II. I precedenti giurisprudenziali e l'orientamento dottrinale prevalente: la tesi del radicale contrasto tra ripudio e ordinamento interno. III. L'emergere di una posizione minoritaria "possibilista" a valle della riforma del sistema italiano di diritto internazionale privato. IV. Quali prospettive per il riconoscimento dei ripudi stranieri? Per un "temperamento" della chiusura in chiave multiculturalista. 


\section{La Cassazione ritorna sul riconoscimento di un ripudio islamico}

1. Con sentenza n. 16804, depositata il 7 agosto 2020, la prima sezione civile della Cassazione è tornata nuovamente sul tema - già affrontato in più occasioni ad opera della giurisprudenza di merito e di legittimità ${ }^{1}$, nonché oggetto di particolari attenzioni da parte della dottrina internazionalprivatistica e non solo - del riconoscimento, in Italia, degli effetti di un provvedimento straniero di ripudio (talaq). Quest'ultimo, come noto, è l'istituto giuridico deputato a realizzare, nel contesto degli ordinamenti giuridici di matrice confessionale islamica, quello stesso scioglimento del vincolo matrimoniale su base volontaria che i sistemi "occidentali", tanto di civil quanto di common law, ricollegano all'istituto del divorzio. Tuttavia, le analogie tra i due istituti si limitano (quasi) esclusivamente a quest'ultimo profilo, posto che assai differenti sono in linea di massima i presupposti, i requisiti per l'accesso alla domanda, i poteri conferiti alle parti e l'iter procedimentale finalizzato alla pronuncia del provvedimento definitivo di scioglimento dell'unione.

2. Il tema è assai complesso se analizzato in chiave comparatistica: a rigore, non esiste un unico istituto del ripudio rintracciabile, sempre uguale a se stesso, all'interno dei vari ordinamenti che attribuiscono valore precettivo alla sharia, ossia alla legge islamica di origine divina. Al contrario vi sono, specie oggigiorno, sensibili differenze tra i connotati che i vari istituti qualificati come talaq assumono nel contesto di ciascun Paese, e nella regolamentazioni delle relazioni familiari tale circostanza è quanto mai evidente e macroscopica: ad alcuni ordinamenti ancorati ad una visione - confessionale ma anche giuridica, posto che i due profili procedono per forza di cose parallelamente - tradizionale e quasi del tutto impermeabile ad ogni tipo di evoluzione normativa o anche solo interpretativa, di cui è l'Arabia Saudita è da tempo perfetto rappresentante, fanno da contraltare altri Paesi che, pur senza disconoscere in alcun modo la matrice sciaraitica del proprio diritto interno, hanno conosciuto nel corso degli ultimi decenni una spiccata "occidentalizzazione", tradottasi nell'approvazione di una regolamentazione dei rapporti familiari fondata su principi quali la piena capacità della donna di succedere e di disporre della propria situazione patrimoniale, la pari dignità dei coniugi nel matrimonio e l'equiordinazione dei genitori nei rapporti con i figli: vale per tutti, su questo secondo fronte, l'esempio offerto dalla Moudawana (il codice riformato del diritto di famiglia) del Marocco ${ }^{2}$.

3. Cionondimeno, in prima approssimazione si può forse affermare che il denominatore comune delle varie forme che l'istituto del ripudio assume nelle esperienze giuridiche degli ordinamenti di matrice islamica ${ }^{3}$ è rappresentato dalla negazione del principio di parità fra $i$ coniugi nel contesto del procedimento che conduce al definito scioglimento del vincolo. Quest'ultimo, infatti, presenta in linea di principio carattere unilaterale e non contraddittorio, quanto meno con riferimento alla fase dell'iniziativa: a ciò può far poi seguito, o meno, una qualche forma di contraddittorio eventuale e differito, laddove sia previsto l'intervento di un primo ripudio non definitivo rispetto al quale è ammessa la possibilità di formulare opposizione, entro un determinato termine, da parte della moglie ripudiata ${ }^{4}$. E si badi - l'istituto del ripudio conserva tali connotati anche laddove ne sia ammessa l'attivazione da parte della donna, alla quale è talora concesso (sulla scorta di uno specifico accordo intercorso in tal senso tra i nubendi/coniugi ${ }^{5}$ ) di "auto-ripudiarsi”" (con o senza l'intervento di un'autorità terza cui presentare

\footnotetext{
${ }^{1}$ Per un'utile rassegna dei precedenti cfr. O. VAnIN, "Ripudio islamico, principio del contraddittorio e ordine pubblico italiano", La nuova giurisprudenza civile commentata, 2015, I, pp. 1031 ss.; C. CAMPIGLIO, "Il diritto di famiglia islamico nella prassi italiana", Rivista di diritto internazionale privato e processuale, 2008, pp. 63 ss.

${ }^{2}$ La versione francese del codice marocchino del diritto di famiglia è reperibile online alla pagina https://adala.justice.gov. $\mathrm{ma} /$ production/legislation/fr/Nouveautes/Code\%20de\%201a\%20Famille.pdf.

${ }^{3} \mathrm{Si}$ noti che esistono altresì ordinamenti di diritto islamico che hanno radicalmente abolito l'istituto del ripudio: è il caso, ad esempio, della Tunisia.

${ }^{4}$ È tuttavia fondamentale evidenziare come normalmente tale opposizione non possa in alcun modo vanificare il ripudio, bensì incida soltanto sul fronte dell'indennizzo economico riconosciuto alla moglie per lo scioglimento dell'unione.

${ }^{5}$ C. Campiglo, "Il diritto di famiglia islamico nella prassi italiana", cit., p. 44, ricorda come il matrimonio, per il diritto islamico classico, sia un vero e proprio contratto, «anzi è uno dei pochi contratti formali previsti da tale diritto».

${ }^{6} \mathrm{Si}$ badi che la titolarità del diritto al ripudio rimane comunque formalmente in capo al marito: è per tale ragione che la concessione di tale facoltà alla donna è normalmente oggetto di specifica pattuizione tra le parti.
} 
la domanda), specie qualora non si preveda che il ripudio concorra con altre forme di scioglimento del vincolo matrimoniale attivabili da parte di entrambi i coniugi.

4. Allo stesso modo, a seconda della specifica regolamentazione nazionale volta a volta considerata, si potrà constatare che l'intero procedimento può aver luogo in forma privata e stragiudiziale o che, al contrario, debba invece essere necessariamente procedimentalizzato con l'intervento di un'autorità pubblica, quanto meno in sede di omologazione dell'atto ${ }^{7}$.

5. Venendo al caso di specie qui considerato, la Cassazione è stata chiamata a confrontarsi con un ripudio pronunciato ai sensi del diritto sciaraitico palestinese, incidente su di un vincolo matrimonio celebrato in Palestina nel 1992 tra un uomo ed una donna aventi, entrambi, doppia cittadinanza giordana ed italiana ${ }^{8}$. Più precisamente, anche alla luce delle maggiori informazioni acquisite dalla Suprema Corte, per il tramite del Ministero della Giustizia, in merito alle norme sostanziali e processuali palestinesi applicate nel procedimento considerato, si è constatato che il procedimento che ha condotto allo scioglimento del matrimonio, seppur fondato sull'applicazione dell'istituto del ripudio, si è concluso con l'adozione di un provvedimento formale ad opera di una pubblica autorità avente natura giurisdizionale nel contesto dell'ordinamento palestinese, ossia il Tribunale di Nablus.

6. La Corte d'appello di Roma, adita dalla donna, con propria sentenza del 12 dicembre $2016^{9}$ aveva accolto la domanda di cancellazione della trascrizione del provvedimento (rectius, dei provvediment $\mathrm{i}^{10}$ ) di scioglimento del matrimonio in ragione della violazione del principio del contradditorio tra le parti, dei diritti di difesa nonché, più in generale, in ragione dell'asserito insanabile contrasto con l'ordine pubblico, con consequenziale impossibilità di riconoscere in Italia il provvedimento straniero in questione ai sensi della legge di riforma del sistema italiano di diritto internazionale privato n. 218 del 31 maggio 1995, art. 64, lett. $b$ e $g$.

7. Con il ricorso per Cassazione da cui la pronuncia in esame trae origine, il marito ha dunque impugnato tale determinazione della Corte d'appello, lamentando (i) l'omessa indagine relativa alla sussistenza dei requisiti per il riconoscimento della sentenza straniera e l'omesso accertamento circa il contenuto della legge straniera applicabile, relativamente tanto all'asserito contrasto con l'ordine pubblico del foro, quanto all'asserita violazione dei diritti di difesa della moglie; (ii) la violazione e falsa applicazione della stessa normativa straniera, sostanziale e processuale, che aveva assunto rilievo nel contesto del procedimento svoltosi nel Paese d'origine. Ad avviso del ricorrente, tali circostanze avrebbero determinato il contrasto con gli artt. 14, 64 lett. $b$ e $g$, nonché 67 della legge 218/1995, rispettivamente dedicati a chiarire che l'accertamento della legge straniera deve essere compiuto d'ufficio da parte del giudice italiano ${ }^{11}$, ad individuare alcuni dei motivi ostativi che possono legittimare il diniego di riconoscimento di una sentenza straniera e, infine, a fissare il procedimento giurisdizionale interno da attivarsi affinché sia per l'appunto accertato in via principale se il provvedimento straniero soddisfa, o meno, i requisiti per il riconoscimento in Italia.

8. Va premesso che non è agevole dedurre, dalla sentenza, la reale portata della doglianza riportata $s u b(i i)$, posto che non è affatto chiaro in quali termini potrebbe essersi realizzata una violazione e/o falsa applicazione del diritto straniero nel contesto di un giudizio interno che dovrebbe vertere es-

\footnotetext{
${ }^{7}$ Quest'ultimo è, ancora una volta, il caso del vigente diritto di famiglia marocchino, come codificato all'interno della Moudawana. Al suo interno il ripudio tradizionale ha lasciato il posto all'istituto qualificato come "divorce sous contrôle judiciaire" (artt. 78 ss.).

${ }^{8}$ Cass. civ., I sez., 7 agosto 2020, n. 16804, in corso di pubblicazione in Rivista di diritto internazionale privato e processuale.

${ }^{9}$ App. Roma, 12 dicembre 2016, in Il diritto di famiglia e delle persone, 2017, p. 347 ss.

${ }^{10} \mathrm{Si}$ tratta infatti di due differenti pronunce rese dal Tribunale di Nablus nel luglio del 2012 (non definitiva) e nel successivo mese di novembre (definitiva).

${ }^{11}$ A norma di quanto prevede l'art. 14 della 1. 218/1995, compendiato nel noto brocardo "iura aliena novit curia".
} 
clusivamente sulla riconoscibilità di un pronuncia resa all'esito di quel giudizio, in ragione degli effetti che essa è in grado di determinare nell'ambito dell'ordinamento richiesto ${ }^{12}$. La decisione, assunta preliminarmente dalla Corte, di esaminare congiuntamente le censure «in quanto connesse», rende tuttavia superflue ulteriori speculazioni a tal riguardo.

9. Le motivazioni in punto di diritto della sentenza appaiono sviluppate secondo un percorso logico non sempre piano e lineare. Dopo aver brevemente richiamato le circostanze di fatto all'origine del provvedimento straniero di ripudio, ed aver sinteticamente tratteggiato gli aspetti salienti dell'istituto per come disciplinato non tanto specificamente dal diritto palestinese applicato nel caso di specie, bensì in termini generali dagli ordinamenti giuridici di matrice confessionale islamica, la sentenza si sofferma sul vigente quadro normativo in tema di riconoscimento di provvedimenti stranieri (extraeuropei), così come tratteggiato dagli artt. 64 ss. della legge 218/1995.

10. L'impressione che si trae dall'analisi della successiva parte motiva è che non sia agevole individuare un preciso percorso argomentativo, a causa di una disordinata giustapposizione di quanti più elementi possibili a sostegno della tesi del diniego di riconoscimento, già sposata dalla Corte d'appello: ad un'ampia rassegna di precedenti giurisprudenziali rispetto ai quali la profonda modificazione del quadro normativo di riferimento in tema di efficacia delle sentenze e degli atti stranieri, avvenuta con la riforma del diritto internazionale privato del 1995, non sembra rivestire particolare significato (la prima pronuncia richiamata risale al 1969, mentre l'ultima è del 2019), si accostano un breve richiamo ai diritti umani incidenti sulle relazioni familiari per come codificati dalla $\operatorname{CEDU}^{13}$; le conclusioni dell'Avvocato generale della Corte di giustizia presentate nel contesto di un procedimento che riguardava un divorzio "privato" siriano ${ }^{14}$ in relazione all'applicazione di due strumenti normativi dell'Unione non rilevanti nel caso di specie ${ }^{15}$; un approfondimento circa la «legge processuale straniera applicabile» (sic; da leggersi forse - anche in considerazione del successivo contenuto - come riferimento al diritto processuale e sostanziale applicato nel caso di specie); e, da ultimo, una succinta digressione di diritto comparato (nel duplice significato di comparazione fra l'istituto del ripudio nei vari ordinamenti islamici nonché di comparazione sull'utilizzo del limite dell'ordine pubblico, in simili contesti, all'interno dei Paesi di area europea, malgrado venga menzionata espressamente solo la Francia) ed una precisazione sui differenti ordinamenti giuridici passibili di trovare applicazione, su base territoriale, nella regione palestinese.

\footnotetext{
${ }^{12} \mathrm{Su}$ quest'ultimo aspetto, cfr. quanto si osserverà più estesamente nei successivi parr.

${ }^{13} \mathrm{Il}$ riferimento è anzitutto agli artt. 8 e 12 della Convenzione, rispettivamente dedicati al diritto al rispetto della vita privata e familiare, il primo, ed al diritto al matrimonio, il secondo, anche ed eventualmente letti in combinato disposto con l'art. 14, teso a sancire il divieto di discriminazione nel godimento dei diritti garantiti dalla Convenzione. Sull'argomento sia consentito il rinvio a F. PESCE, "La tutela europea dei diritti fondamentali in materia familiare: recenti sviluppi", Diritti umani e diritto internazionale, 2016, pp. 5 ss. In questa sede merita di essere richiamata anche l'ulteriore previsione in tema di relazioni familiari di cui all'art. 5 del Protocollo n. 7, volto a sancire e tutelare l'eguaglianza tra i coniugi (come ricorda L. TomasI, "La famiglia nella Convenzione europea dei diritti umani: gli artt. 8 e 14 Cedu", Questione giustizia, n. 2/2019, p. 42, si tratta tuttavia di una norma dal ristretto ambito applicativo e di limitata applicazione giurisprudenziale).

${ }^{14}$ Conclusioni dell'Avv. gen. Saugmandsgaard Øe 14 settembre 2017, Soha Sahyouni c. Raja Mamisch, C-372/16, ECLI:EU:C:2017:686. Si condividono, per inciso, i dubbi sollevati da A. LiCAstro, "La questione della riconoscibilità civile del divorzio islamico al vaglio della Corte di giustizia dell'Unione europea (a margine della pronunzia del 20 dicembre 2017, C-372/16)", Stato, Chiese e pluralismo confessionale, n. 13/2018, www.statoechiese.it, pp. 14-15, in merito alla connotazione realmente privatistica del divorzio sottoposto all'attenzione della Corte di giustizia, atteso che - come nel caso qui in esame - si trattava della pronuncia di un tribunale religioso, equiparato dalla lex fori ad una corte statale ed operante, quindi, sostanzialmente quale organo appartenente all'organizzazione giudiziaria di uno Stato eventualmente anche privo di propri giudici competenti nelle controversie di carattere familiare.

${ }^{15} \mathrm{Si}$ tratta in primis del regolamento (UE) n. 1259/2010 del Consiglio, del 20 dicembre 2010, relativo all'attuazione di una cooperazione rafforzata nel settore del diritto applicabile al divorzio e alla separazione personale (cd. Roma III), GUUE L 343 del 29 dicembre 2010, p. 10; nonché del regolamento (CE) n. 2201/2003 del Consiglio, del 27 novembre 2003, relativo alla competenza, al riconoscimento e all'esecuzione delle decisioni in materia matrimoniale e in materia di responsabilità genitoriale, che abroga il regolamento (CE) n. 1347/2000 (cd. Bruxelles IIbis), GUUE L 338 del 23 dicembre 2003, p. 1.
} 
11. L'insieme delle considerazioni svolte in relazione a tali variegati profili conduce infine la Corte a confermare l'operato della Corte d'appello romana, anche per quanto riguarda il «percorso logico-giuridico» posto alla base della pronuncia, ed a respingere conseguentemente il ricorso, non prima di aver fissato il principio di diritto a tenor del quale «[u]na decisione di ripudio emanata all'estero da una autorità religiosa (...), seppur equiparabile, secondo la legge straniera, ad una sentenza del giudice statale, non può essere riconosciuta all'interno dell'ordinamento giuridico statuale italiano a causa della violazione dei principi giuridici applicabili nel foro, sotto il duplice profilo dell'ordine pubblico sostanziale (violazione del principio di non discriminazione tra uomo e donna; discriminazione di genere) e dell'ordine pubblico processuale (mancanza di parità difensiva e mancanza di un procedimento effettivo svolto nel contraddittorio reale».

12. La decisione, in generale, ed il principio di diritto enucleato dalla Corte, in termini più specifici, offrono l'occasione per operare diverse considerazioni critiche in ordine all'approccio al problema del riconoscimento dei provvedimenti di ripudio ancor oggi condiviso dalla giurisprudenza di legittimità; o, meglio, da una certa - tradizionale - visione che ciclicamente riemerge al suo interno. Come si avrà modo di evidenziare nel prosieguo, infatti, la stessa Sezione prima della Suprema Corte, a distanza di una settimana dalla pronuncia evocata, ha depositato in materia una seconda sentenza, la quale reca considerazioni, ed appare ispirata a principi, in larga misura difformi ${ }^{16}$.

13. Vale la pena di anticipare sin d'ora che, nella decisione sul riconoscimento del ripudio palestinese, ciò che desta maggiori perplessità è rappresentato non tanto dalle conclusioni cui perviene la Corte (ossia l'idea che, nel caso di specie, difettino i presupposti per dare ingresso alla decisione straniera nell'ordinamento interno), quanto dalle argomentazioni in punto di diritto che di tali conclusioni rappresentano il fondamento: qualora si dovesse muovere da esse come imprescindibile riferimento nella gestione di future istanze analoghe, non potrebbe che derivarne un deciso irrigidimento del sistema nel senso della sua chiusura, al punto da rendere ab origine impossibile il riconoscimento degli effetti di ogni ripudio, e ciò a prescindere da qualsiasi valutazione delle circostanze di fatto proprie di ciascuna fattispecie. Così facendo - a parere di chi scrive - si arriverebbe a tradire uno degli stessi principi ispiratori della riforma del sistema interno di diritto internazionale privato, ossia quello in virtù del quale il limite ordine pubblico, stante la sua natura derogatoria rispetto ad una generale apertura all'ingresso delle decisioni straniere, deve trovare applicazione in via del tutto eccezionale, e solo a valle di una verifica relativa agli effetti che la singola pronuncia può determinare nell'ordinamento italiano. Le ragioni di tale affermazione saranno più diffusamente esplicitate nel contesto dei paragrafi seguenti.

\section{I precedenti giurisprudenziali e l'orientamento dottrinale prevalente: la tesi del radicale con- trasto tra ripudio e ordinamento interno}

14. La tesi del contrasto ontologico ed insanabile tra l'istituto islamico del talaq ed i principi irrinunciabili dell'ordinamento italiano è assai risalente e diffusa nel contesto della pronunce che si sono confrontate con il tema della trascrizione di un ripudio straniero ai fini dello scioglimento del vincolo matrimoniale.

15. Anche senza voler rimontare alla posizione espressa dalla Corte d'appello di Roma nel 1948, spesso richiamata ma il cui tenore letterale inevitabilmente tradisce l'appartenenza ad una sensibilità giuridica - impregnata da innegabili pregiudizi - che oramai risulta ampiamente superata ${ }^{17}$, gli stessi

\footnotetext{
${ }^{16}$ Si tratta di Cass. civ., I sez., ordinanza 14 agosto 2020, n. 17170, in corso di pubblicazione in Rivista di diritto internazionale privato e processuale.

${ }^{17}$ In base ad essa, infatti, il ripudio «ripugna alla mentalità morale e giuridica dei popoli che hanno raggiunto un maggior grado di civiltà e che del matrimonio hanno un concetto etico e sociale ben più elevato di quello che ne hanno i popoli orientali» (App. Roma, 29 ottobre 1948, in Foro padano, 1949, I, cc. 348 ss.).
} 
precedenti richiamati dalla prima Sezione della Cassazione nella sua pronuncia qui in esame appaiono orientati, mutatis mutandis, nel senso di affermare un conflitto radicale tra provvedimenti di ripudio e principi fondamentali dell'ordinamento italiano, tale da precludere altresì ogni possibilità di riconoscere effetti giuridici ad un provvedimento di tal sorta già legittimamente pronunciato all'estero in base alla pertinente normativa applicabile.

16. Rispetto a tale orientamento - che potrebbe definirsi consolidato, a voler considerare tanto le pronunce di merito quanto quelle, in realtà esigue ${ }^{18}$, di legittimità - , non paiono aver significativamente inciso né la stessa introduzione dell'istituto del divorzio nell'ordinamento interno, avvenuta con legge $1^{\circ}$ dicembre 1970, n. 898 , né la riforma del sistema di diritto internazionale privato di cui alla già richiamata legge 31 maggio 1995, n. 218.

17. Invero, sul primo fronte, non desta stupore il fatto la mera introduzione dell'istituto del divorzio nel diritto italiano di famiglia non abbia in alcun modo portato con sé una maggiore permeabilità del nostro ordinamento rispetto agli effetti dei ripudi verificatisi nell'ambito di Paesi terzi: non solo si era già osservato in precedenza, nella giurisprudenza interna ${ }^{19}$, come l'avvenuta ratifica della convenzione dell'Aja del 12 giugno 1902 non consentisse di ritenere le pronunce straniere di divorzio come di per sé confliggenti con l'ordine pubblico, qualora ne venisse domandato il riconoscimento in Italia ${ }^{20}$, ma la stessa legge del 1970 si caratterizzava per il fatto di ammettere lo scioglimento del vincolo coniugale esclusivamente al ricorrere di taluni presupposti individuati in via tassativa ${ }^{21}$, sicché la sua entrata in vigore non avrebbe potuto incidere sulla riconoscibilità di provvedimenti volti a sancire tale scioglimento al ricorrere di circostanze radicalmente difformi, come nel caso di una decisione unilaterale e potestativa di ripudio.

18. Venendo al secondo profilo evocato, è agevole rilevare come le argomentazioni addotte in giurisprudenza al fine di impedire ai ripudi islamici di esprimere i propri effetti in Italia siano rimaste - con la sola esclusione di qualche opinione isolata ${ }^{22}$ - pressoché immutate anche a seguito della profonda riforma del sistema di diritto internazionale privato introdotta con la legge 218/1995. Per quanto qui di interesse, tale circostanza desta qualche perplessità non già per il fatto che le corti interne abbiano omesso di adeguare la proprie osservazioni ad un quadro normativo che, sul punto, non era stato interessato da mutamenti radicali ${ }^{23}$; quanto, semmai, per aver (quasi) graniticamente seguitato a condividere considerazioni in tema di riconoscimento dei provvedimenti stranieri che già prima della riforma apparivano superate. Vale infatti la pena di ricordare che, relativamente alla valenza che l'ordine pubblico assume quale limite all'ingresso di provvedimenti stranieri, la legge 218/1995 ${ }^{24}$ si è limitata a meglio esplicitare un elemento che, secondo l'opinione del tutto prevalente in dottrina ${ }^{25}$, già avrebbe dovuto fungere da guida nell'interpretazione dell'art. 797 c.p.c. sino ad allora vigente, dedicato ad indi-

\footnotetext{
${ }^{18}$ Oltre alle due sentenze del 2020 già citate, il riferimento è a Cass. civ., 5 dicembre 1969, n. 3881, in Rivista di diritto internazionale privato e processuale, 1970 , pp. 868 ss.

${ }^{19}$ V. in particolare Cass. civ., 5 dicembre 1969, n. 3881, cit.

${ }^{20} \mathrm{Si}$ tratta della Convenzione dell'Aja del 12 giugno 1902 sui conflitti di leggi e di giurisdizioni in materia di divorzio e di separazione personale, resa esecutiva con 1.7 settembre 1905, n. 523. In base ad essa, «[1]e divorce et la séparation de corps, prononcés par un tribunal compétent aux termes de l'article 5 , seront reconnus partout, sous la condition que les clauses de la présente Convention aient été observées et que, dans le cas où la décision aurait été rendue par défaut, le défendeur ait été cité conformément aux dispositions spéciales exigées par sa loi nationale pour reconnaître les jugements étrangers» (art. 7, par. 1).

${ }^{21}$ Cfr. 1. 898/1970, art. 3.

${ }^{22} \mathrm{Si}$ veda ad es. G. Perlingieri, G. Zarra, Ordine pubblico interno e internazionale tra caso concreto e sistema ordinamentale, Napoli, ESI, 2019, p. 141 ss.; O. VANIN, "Ripudio islamico, principio del contraddittorio e ordine pubblico italiano", cit., spec. p. 1036; C. CAmpiglio, "Identità culturale, diritti umani e diritto internazionale privato", in A. Di Stefano, R. SAPIENZA, $L a$ tutela dei diritti umani e il diritto internazionale, Napoli, Editoriale Scientifica, 2012, spec. pp. 270 ss.

${ }^{23}$ Cfr. N. Boschiero, Appunti sulla riforma del sistema italiano di diritto internazionale privato, Torino, Giappichelli, 1996, spec. p. 154.

${ }^{24}$ Il riferimento è, in particolare, agli artt. 64 e 65.

${ }^{25}$ Cfr. F. Mosconi, C. CAmpiglio, Diritto internazionale privato e processuale. Vol. I. Parte generale e obbligazioni, Milano, UTET Giuridica, $2020^{9}$, p. 394; N. Boschiero, Appunti sulla riforma del sistema italiano di diritto internazionale privato, cit., p. 154; ma in tal senso cfr. già, tra gli altri, E. VITTA, Diritto internazionale privato. I, Torino, UTET, 1972, pp. $406-407$.
} 
viduare le «condizioni per la dichiarazione di efficacia» della sentenza nel quadro del procedimento di delibazione: lo scrutinio di compatibilità non deve operarsi con riguardo alla legge straniera applicata nel giudizio, o con riguardo ai principi giuridici ed ai valori considerati come fondamentali nell'ambito dell'ordinamento a quo, ma - ed esclusivamente - avendo a riferimento gli effetti che il provvedimento straniero, se riconosciuto, è in grado di determinare nell'ordinamento richiesto.

19. Come emerge con evidenza anche dagli stessi precedenti richiamati dalla Suprema Corte nella motivazione della sentenza del 7 agosto 2020 qui in esame, il tema del riconoscimento di provvedimenti stranieri di scioglimento del matrimonio, in generale, e di ripudio, in particolare, è stato quasi costantemente approcciato in termini diametralmente opposti, sia prima che dopo l'entrata in vigore della legge 218/1995. Così, se già nel 1969, in occasione della sua unica pronuncia intervenuta sul tema del talaq, la Cassazione aveva ritenuto legittimo elevare il limite dell'ordine pubblico, innanzi ad un ripudio dichiarato in Iran ${ }^{26}$, non tanto a valle di una valutazione inerente agli effetti che da tale provvedimento potevano derivare all'interno dell' ordinamento italiano, quanto a causa della discriminazione, asseritamente decisiva perché contraria a quell' «uguaglianza morale e giuridica» proclamata dall'art. $29 \mathrm{Cost}^{27}$, tra le posizioni dei due coniugi, ancora nel 2006 la Corte d'appello di Torino ${ }^{28}$, nel richiamarsi incautamente ad un orientamento della giurisprudenza di legittimità non soltanto precedente alla riforma del 1995, ma anche già criticato in dottrina con riguardo al contesto normativo di riferimento ${ }^{29}$, ha esplicitamente proceduto ad un giudizio di compatibilità con l'ordine pubblico dell'istituto del ripudio di diritto islamico: e ciò per il fatto che esso non muove da alcuna delle cause di scioglimento del matrimonio previste dalla legge 898/1970; contrasta con i principi di parità ed uguaglianza tra uomo e donna e con il divieto di discrimi-

${ }^{26}$ Cass. civ., 5 dicembre 1969, n. 3881, cit. Non si era invece posta particolare attenzione sul tema del rispetto dei diritti di difesa della moglie: aspetto che, al contrario, potrebbe ancora oggi risultare dirimente nel vigore della legge 218/1995, e precisamente secondo quanto prescritto dall'art. 64, lett. $b$ e $c$. Secondo quanto può ricavarsi dalla pronuncia, la moglie non aveva partecipato in alcun modo al procedimento che aveva condotto al ripudio, senza però che ne emergano ulteriori dettagli di tutto rilievo: non è dato sapere se la "contumacia" dipendesse da una scelta liberamente operata a valle di una regolare comunicazione relativa al procedimento in corso, né se - fermo restando l'avvio unilaterale dell'iter su impulso, e a discrezione, del solo marito - la moglie avesse una qualche possibilità di opporsi ex post alla relazione degli effetti del ripudio, prima che il provvedimento divenisse definitivo ed irrevocabile (in modo in qualche misura analogo a quanto previsto per i procedimenti monitori nel diritto processuale interno).

${ }^{27}$ Come si evidenzierà nel prosieguo, non solo si è talora sostenuto, anche nella giurisprudenza di legittimità (v. ad es. Cass., 30 settembre 2016, n. 19599, in La nuova giurisprudenza civile commentata, 2017, pp. 362 ss.) che non tutti i principi costituzionali confluiscano all'interno dell'ordine pubblico internazionale (ossia inteso come limite all'ingresso di norme straniere o - come in questo caso - situazioni giuridiche già legittimamente cristallizzatesi all'estero) ma, in ogni caso, il giudizio di compatibilità con tale principio andrebbe più correttamente riferito alle conseguenze che possono derivare dal riconoscimento del provvedimento, e più specificamente alla circostanza che tali conseguenze siano in grado, o meno, di pregiudicare la posizione e gli interessi del soggetto che dall'applicazione del principio di uguaglianza (nel matrimonio) dovrebbe essere tutelato nel caso di specie.

${ }^{28}$ App. Torino, 9 marzo 2006, in Il diritto di famiglia e delle persone, 2007, pp. 156 ss., con nota di A. Sinagra, "Ripudiodivorzio islamico ed ordine pubblico italiano", il quale sposa pienamente le conclusioni cui perviene il collegio e (quasi integralmente) il percorso argomentativo. Si ritiene, al contrario, che siano diverse le criticità rinvenibili nella parte motiva, a partire dalla contestazione relativa all'esercizio della competenza giurisdizionale da parte del foro marocchino ex art. 64, lett. $a$, della 1. 218/1995, e proseguendo con l'asserita violazione dei diritti essenziali della difesa nel procedimento a quo (pur a fronte della mancata comparizione della donna a seguito di una doppia notifica circa l'avvio del procedimento, peraltro contenente la vocatio in ius, ed in assenza di qualsiasi verifica in ordine al fatto che il diritto marocchino applicabile conferisse, o meno, alla moglie, laddove costituita, una reale possibilità di contestare le pretese attoree); con l'osservazione per cui la divergenza tra $\mathrm{i}$ requisiti d'accesso determinerebbe la non sovrapponibilità tra i due istituti (divorzio e ripudio) neppure ai fini dell'invocazione degli effetti di scioglimento del vincolo coniugale; e, infine, con la distinzione tra i concetti di ordine pubblico, in relazione alla quale cfr. amplius la nota seguente.

${ }^{29}$ Il riferimento è a Cass., 13 dicembre 1978, n. 5919, in Il diritto di famiglia e delle persone, 1979, pp. 648 ss.; Cass., 14 gennaio 1982, n. 228, ivi, 1982, pp. 454 ss.; e Cass. $1^{\circ}$ marzo 1983, n. 1539, ivi, 1983, pp. 502 ss. Si tratta della teoria secondo la quale l'ordine pubblico "interno" verrebbe in rilievo con riguardo alle pronunce rese nei confronti dei cittadini italiani, mentre l'ordine pubblico "internazionale", «risultante dai principi comuni alle Nazioni di civiltà affine», dovrebbe considerarsi unicamente nel caso di pronunciate nei confronti di cittadini entrambi stranieri. Cfr. F. MosconI, "Articolo 16", in AA.Vv., Commentario del nuovo diritto internazionale privato, Padova, CEDAM, 1996, pp. 80-81. La corretta distinzione tra ordine pubblico interno ed internazionale, d'altra parte, risultava oramai da tempo pacificamente acquisita in dottrina: si vedano, tra $\mathrm{i}$ tanti, E. VITTA, Diritto internazionale privato. I, cit, pp. 377 ss.; R. QuADRI, Lezioni di diritto internazionale privato, Napoli, Liguori Editore, $1969^{5}$, pp. 361 ss.; R. AGo, Teoria generale del diritto internazionale privato, Padova, CEDAM, 1934, pp. 282 ss. 
nazione in base al sesso per come codificati dalla Costituzione e dalla CEDU; non include «disposizioni a protezione della prole minore» (e dunque si porrebbe altresì in contrasto con l'art. 30, comma 2, Cost.); e persino per il fatto che omette qualsiasi regolazione dei rapporti economici fra i coniugi e, così facendo, si porrebbe altresì in frizione sia con l'art. 29 Cost. che con le disposizioni civilistiche in materia di alimenti e mantenimento. Ora, premesso che la corte torinese sembra aver proceduto nell'individuazione ad abundantiam di ogni possibile criticità e, nel far ciò, aver incluso nella propria rassegna taluni aspetti che senza alcun dubbio non avrebbero dovuto legittimare l'elevazione del limite dell'ordine pubblico ${ }^{30}$, l'aspetto che in questa sede si ritiene meritevole di maggior attenzione è rappresentato dalla circostanza per cui la Corte si è concentrata, con ogni evidenza, sulla valutazione circa la compatibilità con l'ordine pubblico dell'istituto in sé, anziché degli effetti derivanti dal provvedimento con cui si è proceduto ad applicarlo nel caso di specie; per poi concludere, come già anticipato, in termini del tutto negativi.

20. Ebbene, venendo ora alla sentenza n. 16804 del 7 agosto 2020, ne emerge con relativa chiarezza come l'articolato iter argomentativo sottenda ancora una volta la medesima concezione secondo la quale il giudice interno, richiesto del riconoscimento di un provvedimento proveniente da un Paese terzo, sarebbe legittimato a compiere un approfondito esame sulla configurazione - non solo processua$1 \mathrm{e}^{31}$, ma anche sostanziale - di tale ordinamento di per sé considerato, al fine di valutarne la conciliabilità con i principi di diritto ritenuti essenziali ed irrinunciabili dall'ordinamento italiano. Solo in tale ottica si può infatti comprendere non soltanto il disordinato collage di precedenti posizioni giurisprudenziali richiamate nella parte motiva - alcune delle quali esprimevano visioni opinabili già nel proprio contesto temporale di riferimento ${ }^{32}$ - ma anche un tessuto argomentativo da cui tale visione emerge a tratti in modo nitido: nell'evidenziare, ad esempio, la grande varietà di forme che il cd. diritto islamico assume all'interno dei vari ordinamenti fondati sulla sharia, la Corte ritiene che le profonde differenze rinvenibili tra i vari Stati con riguardo alle caratteristiche sostanziali degli istituti, agli organi deputati ad intervenire ed agli aspetti procedimentali siano «tali da poter orientare diversamente il giudizio di compatibilità della legge straniera con l'ordine pubblico» ${ }^{33}$. La stessa richiesta di acquisizione, tramite il Ministero della Giustizia ${ }^{34}$, di dettagliata informativa sulla rilevante normativa materiale palestinese dedicata ai rapporti di famiglia nonché, con specifico riferimento ai diritti di difesa e all'integrità del contraddittorio, parimenti al contenuto della disciplina processuale per come essa risulta formulata nell'ordinamento palestinese (anziché, semmai, quale applicata nel caso concreto ${ }^{35}$ ), sembra ancora una

\footnotetext{
${ }^{30} \mathrm{Si}$ pensi al rilievo per cui il ripudio non presuppone le stesse circostanze di fatto che legittimano lo scioglimento del matrimonio ai sensi della legge 898/1970 (su cui già era intervenuta Cass., 28 maggio 2004, n. 10378, in Rivista di diritto internazionale privato e processuale, 2005, pp. 129 ss., a precisare correttamente come «non possa - di per sé - essere ritenuta contraria all'ordine pubblico italiano una sentenza di scioglimento del matrimonio resa dal giudice straniero fra cittadini italiani facendo applicazione del diritto straniero, per il solo fatto che il matrimonio sia stato sciolto con procedure e per ragioni e situazioni non identiche a quelle contemplate dalla legge italiana»), o a quello della mancata regolazione dei rapporti patrimoniali tra le parti (profilo ben chiarito da Cass., 30 luglio 2012, n. 13556, che molto opportunamente richiama gli esempi delle sentenze non definitive di divorzio e della delibazione delle sentenze ecclesiastiche di nullità del matrimonio concordatario), mentre era semmai il tema della discriminazione tra le parti quello maggiormente idoneo a fondare - in via di principio - la "resistenza" dell'ordinamento interno rispetto all'ingresso del provvedimento di ripudio.

${ }^{31} \mathrm{~A}$ tal proposito potrebbero infatti valere i due limiti ex lett. $b$ e $c$ dell'art. 64 (e ripresi anche nel contesto dell'art. 65 , laddove quest'ultimo richiede il rispetto dei "diritti essenziali della difesa").

${ }^{32}$ Esplicativo, a tal riguardo, è il richiamo alla giurisprudenza inaugurata con la sentenza di Cass. n. 228/1992 e già menzionata $s u b$ nota 29 per quanto attiene alla minoritaria distinzione tra ordine pubblico "internazionale" ed "interno" ivi formulata; vi si afferma, peraltro, che «l'indagine sulla contrarietà o meno di tale statuizione con l'ordine pubblico italiano (...) implica una valutazione delle ragioni della decisione», aprendo dunque, quantomeno apparentemente, ad un riesame nel merito della sentenza resa all'estero di assai dubbia legittimità.

${ }^{33} \mathrm{Sub}$ par. 2.7 (enfasi aggiunta).

${ }^{34}$ Cfr. Cass. civ., I sez., ordinanza interlocutoria $1^{\circ}$ marzo 2019, n. 6161 (reperibile alla pagina http://www.marinacastellaneta.it/blog/la-corte-di-cassazione-sul-riconoscimento-di-un-divorzio-di-natura-privata-pronunciato-da-unistanza-religiosaitalian-court-of-cassation-on-the-recognition-of-a-private-divorce-obtained-before-a-r.html/attachment/6161).

${ }^{35} \mathrm{Si}$ rinvia a quanto si osserverà più estesamente infra, par. IV, per un più ampio esame delle circostanze di fatto a fronte delle quali possa ritenersi soddisfatto quel "rispetto dei diritti della difesa" che viene richiesto sia dall'art. 64 (lett. $b$ e $c$ ) che dall'art. 65 della legge 218/1995, quale requisito ulteriore e distinto rispetto alla mera assenza di contrasto con l'ordine pubblico degli effetti che discendono dal provvedimento straniero in questione.
} 
volta suffragare la generale sensazione che la Suprema Corte abbia fondato il proprio scrutinio di compatibilità con l'ordine pubblico su di un esame relativo alle caratteri propri dell'ordinamento straniero considerato, più che sugli effetti delle decisione di cui si valutava la riconoscibilità.

21. Per inciso, nello sviluppo delle argomentazioni della Corte non pare di grande aiuto - ingenerando semmai qualche incertezza - né il frettoloso richiamo alle previsioni della CEDU (e dei suoi protocolli addizionali) specificamente applicabili ai rapporti tra coniugi e, in termini più generali, al divieto di discriminazione - posto che vi si riconosce come, in effetti, la Corte di Strasburgo non abbia avuto ancora modo di affrontare il tema del riconoscimento di un ripudio islamico nell'ottica precipua della tutela dei diritti umani - né il riferimento alla giurisprudenza Sahyouni della Corte di giustizia ${ }^{36}$. Quest'ultima, in particolare, è riferibile ad una vicenda assai complessa dal punto di vista delle norme di fonte UE che vengono in rilievo ed al relativo ambito di applicazione poiché, più in dettaglio, la Corte di giustizia è stata chiamata a pronunciarsi in via pregiudiziale sull'interpretazione di un regolamento di per sé dedicato ad introdurre norme uniformi sui conflitti di legge in materia di affievolimento e scioglimento del vincolo coniugale, il cui ambito oggettivo di applicazione era stato tuttavia esteso unilateralmente dal legislatore tedesco, al fine di renderlo operativo anche sul fronte del riconoscimento di pronunce straniere (intervenute nella medesima materia della separazione o del divorzio). E non solo. Il carattere peculiare ed irripetibile di quel caso - tale da rendere rischioso qualsiasi tentativo di trarne principi mutuabili in altro contesto - è dato altresì dalla circostanza per cui la Corte ha (frettolosamente, come peraltro evidenziato in dottrina ${ }^{37}$ ) ascritto il provvedimento in questione alla categoria dei "divorzi privati" e, in quanto tale, ha ritenuto che esso esulasse dall' ambito applicativo dello strumento giuridico considerato. Ora, visto che è la stessa Cassazione, poche pagine più avanti, a chiarire come il caso sottopostole non dovesse affatto classificarsi quale divorzio privato ${ }^{38}$, non è chiaro in quale modo le considerazioni svolte dall' Avvocato generale (poiché ampio spazio è riservato proprio all'opinione espressa, nelle proprie conclusioni, da quest'ultimo) e - peraltro - non integralmente confluite nella pronuncia della Corte, in relazione ad uno strumento che non si applica al riconoscimento dei divorzi stranieri (se non in Germania, a valle di una scelta discrezionale di tale ordinamento, e comunque con alcuni limiti ${ }^{39}$ ) e che, comunque, contiene previsioni autonome (e da interpretarsi autonomamente rispetto agli ordinamenti nazionali) in tema di restrizioni all'applicazione del diritto straniero richiamato, possano essere utilmente trasposte al caso in esame ${ }^{40}$.

22. Tornando, invece, alle riflessioni svolte dalla Suprema Corte con riguardo al riconoscimento delle pronunce straniere in Italia ai sensi della legge 218/1995, ossia al tema qui centrale, va anzitutto

\footnotetext{
${ }^{36}$ Si tratta, oltre che delle conclusioni dell'Avv. gen. già dettagliate in nota 14, della sentenza della Corte di giustizia 20 dicembre 2017, Soha Sahyouni c. Raja Mamisch, C-372/16, ECLI:EU:C:2017:988; nonché dell'ordinanza della Corte di giustizia 12 maggio 2016, Soha Sahyouni c. Raja Mamisch, C-281/15, ECLI:EU:C:2016:343.

${ }^{37}$ Cfr. A. Licastro, "La questione della riconoscibilità civile del divorzio islamico al vaglio della Corte di giustizia dell'Unione europea (a margine della pronunzia del 20 dicembre 2017, C-372/16)", cit., spec. pp. 14 ss.

38 «La compagine strutturale, pur nella sua peculiarità, sembra dunque essere quella di un tribunale religioso avente comunque funzioni giurisdizionali in determinate materie, con la conseguenza che alla decisione non può attribuirsi la natura di mero divorzio privato pronunciato da organi non inseriti nella compagine giurisdizionale» (par. 2.8).

${ }^{39}$ In questo senso si ritiene dubbia la tesi, espressa dall' Avv. gen., secondo cui «non è ammissibile far variare l'interpretazione dell'art. 10 del regolamento n. 1259/2010 a seconda che la causa verta su una domanda di divorzio, caso normale di applicazione di tale regolamento, nel quale sarebbe sufficiente l'esistenza di una discriminazione astratta, oppure sul riconoscimento di una decisione di divorzio, caso di applicazione del medesimo risultante dal diritto tedesco, nel quale sarebbe necessario l'accertamento di una discriminazione concreta». Infatti, fermo restando che l'uniformità interpretativa delle norme di diritto dell'UE può essere garantita dalla Corte di giustizia anche al di là dei limiti rappresentati dal rispettivo ambito di applicazione (ad esempio laddove - come nel caso di specie - una prassi nazionale si consolidi nel senso di estendere unilateralmente l'ambito applicativo di uno strumento di diritto dell'UE: cfr. ex multis sentenza della Corte di Giustizia 18 ottobre 2012, Nolan, C-583/10, ECLI:EU:C:2012:638, punto 46), vale la pena di evidenziare che, conformemente al $\S 109$ del Gesetz über das Verfahren in Familiensachen und in den Angelegenheiten der freiwilligen Gerichtsbarkeit (FamFG), l'ordinamento tedesco risulta provvisto di propri ed autonomi motivi ostativi al riconoscimento delle decisioni straniere in materia matrimoniale.

${ }^{40}$ «Se si ritiene che l'impostazione della Corte di giustizia sia applicabile al caso in esame, si può fondatamente dubitare che il ripudio costituisca "provvedimento" ai sensi dell'art. 65 1. cit., giacché rimane un atto di privata volontà, sia pure reso davanti ad un'autorità religiosa locale, che si limita ad autenticarlo» (par. 2.5).
} 
evidenziato che la decisione individua nell'art. 65 la norma tesa a regolare il riconoscimento del provvedimento palestinese di ripudio sottoposto al suo giudizio. Sotto questo profilo, non paiono condivisibili né l'opinione, parimenti espressa dal collegio, per cui risulterebbe «esclusa la generale applicabilità dell'art. 64, poiché il ripudio può non conseguire ad un atto assimilabile ad una sentenza» (è infatti relativamente pacifica, in dottrina, l'alternatività tra i due percorsi a fronte di provvedimenti che ricadano nell'ambito applicativo dell'art. 65 , atteso che la portata di quest'ultimo è più ristretta ed interamente compresa all'interno di quella dell'art. 64), né il conseguente riferimento all'art. 65 della legge 218/1995. Vero è, infatti, che quest'ultima norma ha ad oggetto i provvedimenti ${ }^{41}$ stranieri «relativi alla capacità delle persone nonché all'esistenza di rapporti di famiglia o di diritti della personalità»; ma è altrettanto vero che, in base al suo inequivoco tenore letterale, è altresì necessario che tali provvedimenti provengano dallo Stato la cui legge è richiamata dalle pertinenti norme di conflitto dell'ordinamento italiano ${ }^{42}$. E se il primo requisito appare senz'altro soddisfatto nel caso di specie, lo stesso non sembra potersi dire con riguardo al secondo: in base a quanto previsto dall'art. 31 della stessa legge 218/1995, la separazione personale e lo scioglimento del matrimonio sono regolati dalla legge nazionale comune dei coniugi al momento della domanda. Ora, poiché all'introduzione del giudizio entrambi i coniugi risultavano possedere doppia cittadinanza giordana ed italiana, e poiché la legge 218/1995 dedica a tale eventualità un'apposita previsione invero oggetto di critiche sin dalla sua approvazione ${ }^{43}-$ a tenor della quale, in caso di cittadinanze plurime all'interno delle quale figuri la cittadinanza italiana, quest'ultima prevale, deve ritenersi che lo Stato la cui legge è richiamata dal diritto internazionale privato italiano sia, per l'appunto, l'Italia ${ }^{44}$. Ne discende che, in questo caso, l'art. 65 non consentirebbe di individuare in un Paese straniero l'ordinamento a partire dal quale garantire il riconoscimento "privilegiato" dei provvedimenti per il tramite delle norme di conflitto.

23. È pur vero, tuttavia, che il tema della scelta tra i due percorsi offerti dagli artt. 64 e 65 della legge 218/1995 non assume, in concreto, un rilievo determinante per quanto d'interesse in questa sede, poiché i limiti all'ingresso di provvedimenti stranieri rappresentati dal contrasto con l'ordine pubblico e dalla violazione dei diritti essenziali della difesa risultano comuni ad entrambe le previsioni ${ }^{45}$, sicché analoghe sono le riflessioni che possono svolgersi a tal riguardo.

24. Nel contesto della sentenza depositata il 7 agosto 2020 risulta difficile enucleare con precisione i singoli e specifici vizi del provvedimento straniero in ragione dei quali viene condivisa la posizione espressa dalla Corte d'appello, nel senso del rigetto del suo riconoscimento. Ancora una volta, infatti, la Suprema Corte sembra procedere con qualche incertezza: ne è riprova il fatto che l'individuazione, nel caso di specie, di uno, o entrambi, i motivi di rifiuto del riconoscimento a norma dell'art. 65 sembra effettuata tramite la giustapposizione di una serie di considerazioni le quali dovrebbero condurre, tutte assieme, a fugare ogni dubbio circa la correttezza delle conclusioni cui perviene il collegio. Infatti, dopo aver chiarito - come già anticipato - che il provvedimento de quo non sembra potersi ascrivere alla categoria dei cd. divorzi privati, la Cassazione individua anzitutto una serie di norme costituzionali

\footnotetext{
${ }^{41}$ È appena il caso di ricordare che non vi è unanimità, in dottrina, nel ritenere (secondo la tesi accolta, tra gli altri, da S. Bariatti, Articolo 65, in Aa.Vv., Commentario del nuovo diritto internazionale privato, cit., pp. 332-333; N. BoschiERo, Appunti sulla riforma del sistema italiano di diritto internazionale privato, cit., p. 164) che l'art. 65 si riferisca ad un novero di provvedimenti più ampio rispetto a quello delle sole sentenze: cfr. in particolare P. Picone, La riforma italiana del diritto internazionale privato, Padova, CEDAM, 1998, pp. 483 ss.

${ }^{42}$ Posto che l'art. 65 fa testualmente riferimento allo «Stato la cui legge è richiamata dalle norme della presente legge» (enfasi aggiunta), deve ritenersi che il richiamo debba intendersi operato all'art. 31 della medesima 1. 218/1995 (pure oramai privo di residuo ambito applicativo, poiché da disapplicarsi in toto a vantaggio del regolamento n. 1259/2010, che prevale su di esso): cfr. F. Mosconi, C. CAmpiglio, Diritto internazionale privato e processuale. Vol. I. Parte generale e obbligazioni, cit., p. $399-400$.

${ }^{43}$ Cfr. per tutti A. DAvì, "Le questioni generali del diritto internazionale privato nel progetto di riforma", in G. GAJA (a cura di), La riforma del diritto internazionale privato e processuale, Milano, Giuffrè, 1994, pp. 88 ss.

${ }^{44} \mathrm{Né} \mathrm{si} \mathrm{potrebbe} \mathrm{pervenire} \mathrm{a} \mathrm{diversa} \mathrm{conclusione} \mathrm{anche} \mathrm{a} \mathrm{voler} \mathrm{ritenere} \mathrm{applicabile} \mathrm{l'art.} 8$ del regolamento 1259/2010 al posto dell'art. 31 della 1. 218/1995 (vd. nota 42), poiché la residenza abituale delle parti (principale tra i criteri di collegamento valorizzati dal regolamento per il caso di mancata optio legis) risulta ugualmente localizzata in Italia.

${ }^{45}$ Come condivisibilmente già notava $\mathrm{O}$. VANIN, "Ripudio islamico, principio del contraddittorio e ordine pubblico italiano", cit., spec. p. 1036.
} 
e/o internazionali pattizie che, andando ad integrare principi di ordine pubblico internazionale ${ }^{46}$, risulterebbero ostative rispetto al riconoscimento richiesto, atteso che quest'ultimo non potrebbe avvenire se non pregiudicandone irrimediabilmente l'osservanza. Il riferimento all'ordine pubblico processuale rappresenta, poi, l'occasione per un breve passaggio in cui si evidenziano le circostanze di fatto che risulterebbero lesive dei diritti essenziali di difesa della moglie, peraltro rimasta contumace nel contesto del procedimento conclusosi con il ripudio irrevocabile.

25. Tuttavia a destare le maggiori perplessità sono forse le considerazioni espresse in chiusura della parte motiva ove, nell'evidente intento di suffragare ulteriormente il giudizio di non riconoscibilità del provvedimento richiesto, non solo si afferma che il mancato accertamento, da parte del tribunale sciaraitico, dell' «effettiva cessazione del rapporto affettivo e di convivenza dei coniugi ovvero della possibilità di una sua composizione e continuazione» risulterebbe lesivo di un principio di ordine pubblico processuale ( $\mathrm{sic}$ ) e che, con riferimento all'ordine pubblico sostanziale, l'«istituto del ripudio» di cui al diritto giordano (applicato in Palestina) risulterebbe discriminatorio per la donna, poiché fondato su di una decisione unilaterale e potestativa del solo marito, andando così ad integrare una valutazione sulla riconoscibilità operata assolutamente ex ante ed in astratto. In aggiunta a ciò, infatti, la Corte ritiene opportuno fissare un principio di diritto che sembra radicalmente smentire lo spirito cui è ispirato il riconoscimento delle decisioni e, più in generale, dei provvedimenti stranieri in Italia, quantomeno a partire dalla riforma del sistema di diritto internazionale privato del 1995: anche nel caso in cui il ripudio sia sancito attraverso una pronuncia equiparabile ad una sentenza (poiché resa da un'autorità che, seppur non statale, risulta investita di funzioni giurisdizionali effettive ad opera dell'ordinamento considerato), «una decisione di ripudio (...) non può essere riconosciuta all'interno dell'ordinamento giuridico statuale italiano a causa della violazione dei principi giuridici applicabili nel foro, sotto il duplice profilo dell'ordine pubblico sostanziale (violazione del principio di non discriminazione tra uomo e donna; discriminazione di genere) e dell'ordine pubblico processuale (mancanza di parità difensiva e mancanza di un procedimento effettivo svolto nel contraddittorio reale) $\rangle^{47}$.

26. Abbandonando, dunque, il richiamo ad ogni possibile violazione dei diritti essenziali della difesa e/o del diritto al contraddittorio quali possibili vizi a sé stanti (in quanto codificati in via autonoma - come si è visto - sia dall'art. 64, lett. $b$ e $c$, che dall'art. 65 della legge 218/1995), la Cassazione opta per la fissazione di un principio rigido ed impermeabile a qualsiasi valutazione circa le specificità del singolo provvedimento volta a volta considerato: il ripudio sarebbe di per sé incompatibile con l'ordine pubblico internazionale dell'ordinamento italiano, sotto il duplice profilo indicato. Il corollario che discende da una posizione di tal sorta appare, a questo punto, ineludibile: non v'è ragione per sottoporre a valutazione, caso per caso, i provvedimenti di ripudio legittimamente resi nel contesto di ordinamenti stranieri di matrice islamica, al fine di vagliarne la riconoscibilità in Italia, poiché la radicale incompatibilità dell'istituto con l'ordine pubblico del foro è sancita dalla Cassazione con una valutazione compiuta, ex ante, una volta per tutte. È fin scontato evidenziare come una valutazione di tal sorta abbia ad oggetto, inevitabilmente, le (sole) caratteristiche proprie dell'istituto di diritto straniero e del procedimento teso alla sua adozione.

27. E va da sé che, sulla scorta delle considerazioni sin qui sinteticamente compendiate, il giudice di legittimità conclude quindi per il rigetto del ricorso avverso la pronuncia della Corte d'appello di Roma e la conseguente conferma della non riconoscibilità del provvedimento palestinese in oggetto. Come si avrà modo di evidenziare più diffusamente nel prosieguo, la scelta del rigetto di cui al dispositivo pare, forse, l'aspetto della sentenza in oggetto che desta le minori perplessità; ma tale conclusione avrebbe potuto essere ugualmente raggiunta attraverso un percorso argomentativo maggiormente fedele ai principi del diritto internazionale privato vigente.

\footnotetext{
${ }^{46} \mathrm{Si}$ tratterebbe, più nel dettaglio, degli artt. 2, 3, 29 e 111 Cost.; degli artt. 6 e 14 CEDU; dell'art. 5 del Protocollo n. 7 alla CEDU; dell'art. 16 della Convenzione ONU del 1979 sull'eliminazione di ogni forma di discriminazione nei confronti delle donne (CEDAW).

${ }^{47}$ Enfasi aggiunta.
} 


\section{L'emergere di una posizione minoritaria "possibilista" a valle della riforma del sistema italia- no di diritto internazionale privato}

28. A completamente di quanto sin qui osservato con particolare riguardo alla sentenza del 7 agosto 2020 resa dalla Prima sezione della Corte di cassazione, si rende tuttavia necessario dar atto della circostanza per cui, anche nel contesto della riflessione dottrinale, le voci a supporto di una generalizzata "chiusura" dell'ordinamento interno rispetto all'ingresso di provvedimenti di ripudio sembrano essere state fino ad oggi maggioritarie.

29. Partendo dall'oggettiva, e profonda, distanza che sussiste fra l'istituto islamico del talaq e quello del divorzio oramai rinvenibile all'interno di tutti gli ordinamenti giuridici europei, anche la dottrina più attenta, infatti, ha espresso riserve in ordine alla possibilità che gli effetti di un provvedimento di ripudio possano trovare riconoscimento al di fuori del Paese di origine ${ }^{48}$; ciò, per svariate ragioni inerenti all'insanabile contrasto tra le caratteristiche immanenti all'istituto straniero in questione ed i principi su cui poggia la possibilità di ottenere lo scioglimento del matrimonio per divorzio - in Italia così come negli altri Paesi di civil e common law ${ }^{49}$ - nonché sugli stessi caratteri propri dell'istituto del ripudio, sovente ritenuti di per sé inaccettabili ed odiosi $i^{50}$. Una posizione analoga, peraltro, è rinvenibile anche nel contesto della dottrina ${ }^{51}$ e della più recente giurisprudenza ${ }^{52}$ di un ordinamento europeo, qual è quello francese, particolarmente sensibile al problema del riconoscimento degli effetti del ripudio, in ragione del rilevante fenomeno migratorio che origina dall'area francofona nordafricana e di cui la Francia è tradizionalmente destinataria ${ }^{53}$.

${ }^{48}$ Cfr. R. Clericl, "La compatibilità del diritto di famiglia mussulmano con l'ordine pubblico internazionale", Famiglia e diritto, 2009, pp. 197 ss., spec. p. 202.

${ }^{49} \mathrm{Si}$ sono così valorizzati, volta a volta, il generale divieto di discriminazione in base al sesso, il principio di uguaglianza tra uomo e donna all'interno del matrimonio, i diritti fondamentali della difesa ed il connesso diritto al contraddittorio, ecc. Con più specifico riguardo all'ordinamento italiano, si sono talora evidenziate ulteriori criticità inerenti alla specifica configurazione del divorzio nell'ambito della legge 898/1970: su tutte, vale la pena di ricordare la tesi per cui rientrerebbe all'interno del nostro ordine pubblico internazionale il divieto di scioglimento del matrimonio ad iniziativa libera ed immotivata di un coniuge o, più in generale, in assenza di un previo accertamento circa l'impossibilità di mantenere o ricostituire la «comunione materiale e spirituale tra i coniugi», rinvenibile sia in dottrina (si vedano ad es. le osservazioni svolte - seppur indirettamente poiché non riferite alla dimensione internazionalprivatistica del divorzio - da C. IRTI, "Gestione condivisa della crisi familiare: dalla mediazione familiare alla negoziazione assistita”, Il diritto di famiglia e delle persone, 2016, spec. pp. 677 ss.; F. TOMMASEO, "La gestione dei conflitti coniugali tra autonomia privata e giurisdizione", Famiglia e diritto, 2015, spec. pp. 1055 ss.) che nella stessa giurisprudenza di legittimità (cfr. per tutte Cass., 28 maggio 2004, n. 10378, cit.). È appena il caso di evidenziare come una censura di tal ultima sorta non potrebbe essere egualmente mossa ad opera delle corti di quei Paesi che, nel tempo, hanno aderito ad una concezione del matrimonio di stampo maggiormente "contrattualistico" rispetto a quella degli ordinamenti europei di area mediterranea, posto che tale concezione generalmente si accompagna con la facoltà di scioglimento del vincolo coniugale su base meramente consensuale. Peraltro, a seguito dell'introduzione, con il d.1. 132/2014 (convertito in 1. 162/2014), dei due nuovi percorsi offerti dalla negoziazione assistita e dall'accordo concluso innanzi all'ufficiale di stato civile, non sembra irragionevole sostenere che, anche in Italia, i tradizionali requisiti soggettivi rappresentati dalla cessazione della comunione materiale e spirituale tra i coniugi, prescritti affinché possa essere pronunciato lo scioglimento del vincolo matrimoniale, stiano rapidamente perdendo ogni significato residuo. (contra P. VIRGADAMO, "Ripudio islamico e contrarietà all'ordine pubblico tra unitarietà del limite e corretta individuazione dei principi", Il diritto di famiglia e delle persone, 2017, p. 362, nota 43). È stato altresì osservato come il sempre più agevole riconoscimento di sentenze estere per mutuo consenso possa spingere in direzione del riconoscimento di ripudi con donna consenziente: cfr. S.A. Aldeeb Abu-SAhlien, "Droit musulman de la famille et des successions en Suisse", Revue critique de droit international privé, 2007, p. 519.

${ }^{50}$ Come già evidenziato, un giudizio di questo tipo tende a discendere da uno scrutinio operato essenzialmente in astratto. Si tratta della posizione sposata, ad es., da M.-L. NiвоYет, "Regard français sur la reconnaissance en France des répudiations musulmanes", Revue internationale de droit comparé, 2006, pp. 27 ss., che infatti segnala i rischi che a suo avviso discendono dallo spingere «jusqu'à l'absurde le principe de l'appréciation in concreto» al posto di quella «portée sur le contenu de la loi étrangère» (p. 32).

${ }^{51}$ Cfr., tra gli altri, M.-E. Ancel, "The New Policy of the Cour de Cassation Regarding Islamic Repudiations. A Comment on Five Decisions Dated 17 feb. 2004", Yearbook of Private International Law, 2005, pp. 261 ss.

${ }_{52}$ Il revirement della giurisprudenza francese viene normalmente ricondotto alle pronunce della Cour de cassation del 17 febbraio 2004, in Revue critique de droit international privé, 2004, p. 423 ss., con nota di P. Hammse. Nello stesso senso cfr. altresì, successivamente, Cour de cassation, 4 novembre 2009, ivi, 2010, p. 369 ss., ed il commento di K. ZAHER, "Plaidoyer pour la reconnaissance des divorces marocains", ibidem, pp. 313 ss.

${ }^{53}$ È stato evidenziato (R. CLERICI, "La compatibilità del diritto di famiglia mussulmano con l'ordine pubblico internaziona- 
30. Ed è ancora la stessa chiusura "aprioristica" nei confronti dei provvedimenti stranieri di ripudio a potersi rintracciare, in Italia, anche al di fuori della giurisprudenza e della letteratura già richiamate: anche in ragione del ruolo necessario e preliminare svolto dagli ufficiali di stato civile con riguardo alla trascrizione dei provvedimenti stranieri di scioglimento del vincolo matrimoniale, non può infatti dimenticarsi di evidenziare come all'interno del Massimario per l'ufficiale di stato civile predisposto dal Ministero dell'Interno si rinvenga un titolo espressamente dedicato al (riconoscimento di una pronuncia straniera di) ripudio, ove, in modo assai tranchant, si afferma che «[i]l procedimento indicato come "atto di ripudio" rappresenta una fattispecie contraria all'ordine pubblico e, in sostanza, in contrasto con la lettera g) dell'art. 64 della legge 218/1995» $\aleph^{54}$. Anche in questo caso, la valutazione è dunque condotta in termini squisitamente astratti, attraverso un giudizio che, formulato una volta per tutte, sembra precludere pro futuro qualsiasi tentazione di procedere a scrutini individualizzati ${ }^{55}$. Alla luce di simili premesse, appare quasi contraddittoria la successiva precisazione per cui «[p]eraltro, la questione può essere rimessa, da parte di chiunque vi abbia interesse, alla valutazione della competente corte d'appello»: la ragione che ha condotto ad introdurla può, forse, essere individuata proprio in una sottostante volontà di temperare quel radicale giudizio di incompatibilità con l'ordinamento italiano formulato ex ante, allo stesso tempo devolvendo ai competenti organi giurisdizionali il delicato compito di valutare se e quando possa essere ammessa la trascrizione di un ripudio.

31. Fermo restando che, dunque, la tesi dell' "incompatibilità ontologica" pare ancor oggi (ed anzi soprattutto oggi, almeno nell'esperienza francese) raccogliere ampi favori, diffusi in modo trasversale tra la prasi giudiziaria e la riflessione teorica, deve altresì darsi conto di alcune voci di segno differente.

32. Come già si è anticipato, prima del più recente revirement con cui si sono sostanzialmente chiuse le porte all'ingresso di provvedimento stranieri di ripudio, già la giurisprudenza francese aveva conosciuto un orientamento di disponibilità al riconoscimento, seppur solo in determinati casi ed a valle di un rigoroso scrutinio circa le specifiche peculiarità di ogni richiesta, nell'ottica di garantire ai cittadini stranieri, residenti in Francia, il riconoscimento dello status libero legittimamente acquisito all'estero ${ }^{56}$; analogamente, è altresì possibile rinvenire, ad esempio, taluni precedenti favorevoli al riconoscimento da parte delle autorità pubbliche svizzere ${ }^{57}$ e della giurisprudenza tedesca ${ }^{58}$.

le", cit., p. 203) come tale orientamento sia fortemente avallato da alcuni Autori provenienti da Paesi islamici: cfr. ad es. M.-C. NAJM, "Le sort des répudiations musulmanes dans l'ordre juridique français. Droit et idéologie(s)", Droit et cultures, 2010/1, pp. 209 ss.; A. MezGHani, "Quelle tolérance pour les répudiations?", Revue internationale de droit comparé, 2006, pp. 61 ss.; ID., "Le juge français et les institutions du droit musulman", Clunet, 2003, pp. 721 ss. La ragione pare essere di stampo didascalico e pedagogico, risiedendo nell'obiettivo di promuovere, seppur indirettamente, il ricorso a forme differenti di divorzio, al tempo stesso incentivando prassi maggiormente compatibili con la tutela dei diritti fondamentali della donna (in questi termini, con specifico riferimento al caso dei cittadini marocchini, anche M.-L. NiBoyet, "Regard français sur la reconnaissance en France des répudiations musulmanes", cit., pp. 27 ss.). Come si evidenzierà nel prosieguo, l'eventuale e possibile sacrificio di interessi individuali (della donna, a veder riconosciuto il proprio stato libero) in tale prospettiva non pare del tutto convincente.

${ }^{54}$ Massimario per l'ufficiale di stato civile (ediz. 2012), sub 11.3.1. (pag. 148).

${ }^{55}$ A maggior riprova di ciò, e per fugare ogni eventuale dubbio, poco più avanti ci si preoccupa di chiarire che è proprio «l'istituto in quanto tale che risulta in contrasto con il nostro ordinamento e con principi inderogabili di ordine pubblico» (ibidem).

${ }^{56}$ Cour de Cassation, İ̀re Sect., 3 novembre 1983, Rhobi, in Revue critique de droit international privé, 1984, pp. 325 ss., con nota di I. Fadlallah. Ma, più di recente, cfr. ancora Cour de Cassation, I İre Sect., 3 luglio 2001, Douibi, in Revue critique de droit international privé, 2001, pp. 704 ss., con nota di L. GANNAGÉ, ed in relazione alla quale cfr. altresì M.-L. NiBOYET, in Recueil Dalloz, 2001, pp. 3378 ss.

${ }^{57}$ Cfr. Dipartimento dell'Interno del Canton Argovia, decisione del 4 maggio 1971, in Schweizerische Juristen-Zeitung, 69/1973, p. 25. Sul punto v. amplius S. AldeEB, A. Bonomi (eds.), Le droit musulman de la famille et des successions à l'épreuve des ordres juridiques occidentaux, Zürich, Schulthess Polygraphischer Verlag,1999, pp. 207 ss.

${ }^{58}$ Queste ultime, in particolare, sembrano aver valorizzato l'Inlandsbeziehung della fattispecie dedotta in giudizio con l'ordinamento del foro (si tratta di un'idea sostanzialmente corrispondente a quella di ordine pubblico di prossimità): cfr. ad es. AG Hamburg, 24 gennaio 1985, in IPRax 1986, pp. 114 ss., con nota di D. HenRich. Sul punto v. ancora S. AldeEB, A. BonOMI (eds.), Le droit musulman de la famille et des successions à l'épreuve des ordres juridiques occidentaux, cit., pp. 190-191. 
33. L'ordinamento italiano, al contrario, è stato a lungo caratterizzato da una giurisprudenza di merito ${ }^{59}$ saldamente allineata, nel senso di una totale chiusura, con il pur isolato orientamento espresso dalla Corte di cassazione nel $1969^{60}$, ossia laddove quest'ultima aveva avuto modo di confrontarsi direttamente con lo specifico tema dell'attribuzione di effetti nel foro ad un ripudio pronunciato in un Paese terzo. Nondimeno, dopo che l'opportunità di un approccio differente e più elastico al tema era già stato auspicato in dottrina ${ }^{61}$, un rilevante segnale di discontinuità è stato offerto da una sentenza del 2008 con cui la Corte d'appello di Cagliari ha dichiarato l'efficacia di un «provvedimento di divorzio» ${ }^{62}$ egiziano e, per l'effetto, ne ha ordinato la trascrizione nei registri di stato civile italiani ${ }^{63}$. La fattispecie dedotta all'attenzione del collegio cagliaritano, invero, presentava alcune peculiarità obiettivamente favorevoli alla riconoscibilità del provvedimento straniero e che meritano di essere evidenziate: se infatti, da un lato, la moglie risultava non aver prestato il proprio consenso (seppur ex post, a seguito di notifica, ritualmente avvenuta, del primo talaq revocabile) allo scioglimento dell'unione, dall'altro lato era emerso dagli atti come, una volta che (a valle del prescritto periodo di "ritiro legale" - detto 'iddateso a verificare l'assenza di una gravidanza in atto) il divorzio era divenuto irrevocabile, non soltanto ella aveva ottenuto la parte residua della propria dote e l'intera compensazione economica prevista dalla legge egiziana per il caso di mancato assenso al ripudio (mut'ah, o "indennità di consolazione"), ma era addirittura convolata a nuove nozze; così avvalendosi, in concreto, degli effetti del ripudio. E venendo al giudizio sul riconoscimento di cui è stata investita la Corte d'appello di Cagliari, occorre altresì evidenziare sia il fatto che la controversia aveva avuto origine non dall'opposizione della (ex) moglie, bensì dal rifiuto opposto alla richiesta di trascrizione del divorzio, formulata dall'attore, da parte dell'ufficiale di stato civile; sia la circostanza per cui la donna era stata dichiarata contumace solo dopo esser stata ritualmente invitata a costituirsi in giudizio, con notifica di cui si era verificato il buon fine. A ciò deve poi aggiungersi che, dopo il ripudio, l'uomo aveva trasferito la propria residenza in Italia, acquisito (anche) la cittadinanza italiana ed infine contratto, in Egitto, un nuovo matrimonio, da cui erano nati quattro figli; la donna, al contrario, aveva mantenuto la propria residenza all'estero.

34. Si trattava, dunque, di una situazione del tutto particolare in cui è difficile escludere che la Corte, pur formalmente ancorando le proprie motivazioni su di un asserito rispetto del principio del contraddittorio tra le parti e dei diritti fondamentali della difesa, abbia in realtà compiuto anzitutto una valutazione - di ordine estremamente concreto e fattuale - circa gli effetti che nel caso specifico potevano derivare dal riconoscimento (o dal mancato riconoscimento) del talaq egiziano. In altri termini, si tratta di una fattispecie a fronte della quale l'attribuzione di effetti nel foro al provvedimento straniero avrebbe determinato un indubbio beneficio al richiedente senza parallelamente arrecare alcun pregiudizio alla posizione della convenuta; e, al contempo, dal rigetto della domanda di riconoscimento sarebbero potuti derivare unicamente conseguenze pregiudizievoli a carico di entrambi gli ex coniugi, i quali non si sarebbero visti riconoscere dall'ordinamento italiano quello stesso status familiare su cui

\footnotetext{
${ }^{59} \mathrm{~V}$. ad es., già in precedenza, Corte d'appello di Milano, 14 dicembre 1965, in Rivista di diritto internazionale privato e processuale, 1966, pp. 381 ss.; nonché, successivamente, Corte d'appello di Roma, 9 luglio 1973, in Il diritto di famiglia e delle persone, 1974, pp. 653 ss.; Corte d'appello di Trieste, 23 ottobre 1980, in Rivista di diritto internazionale privato e processuale, 1982, pp. 395 ss.

${ }^{60}$ Cass. civ., 5 dicembre 1969, n. 3881, cit.

${ }^{61} \mathrm{Si}$ veda già C. CAmpiglio, "Il diritto di famiglia islamico nella prassi italiana", cit., p. 66: «[d]al momento che il limite dell'ordine pubblico ha la funzione di impedire la produzione in Italia - da parte di norme o sentenze straniere - di "effetti" contrari ai nostri principi fondamentali, il suo intervento dovrebbe essere vagliato caso per caso, e ammesso solo in via eccezionale. La tendenza giurisprudenziale a farlo scattare sistematicamente, ogniqualvolta di discuta di ripudio, ne tradisce la ratio»».

${ }^{62}$ Così, testualmente, il dispositivo della sentenza. Tuttavia, come emerge chiaramente dalla precedente motivazione, si tratta di un vero e proprio ripudio ottenuto tramite la pronuncia, da parte del marito, della formula tradizionale del talaq.

${ }^{63}$ Corte d'appello di Cagliari, 16 maggio 2008, in Rivista di diritto internazionale privato e processuale, 2009 , pp. 647 ss. Per un commento cfr. A. MARIGNANI, "Est-il encore possible de reconnaître des répudiations prononcées à l'étranger? - L'étude comparée de la transposition possible d'une solution italienne à la France", Clunet, 2013, pp. 458 ss., la quale, auspicando che la stessa giurisprudenza francese possa intraprendere un percorso analogo e temperare così le rigide posizioni espresse dalla Cassation a partire dal 2004, evidenzia in particolare, circa il possibile rilievo della teoria dell'ordine pubblico di prossimità nel caso di specie, come (a differenza della totalità dei casi sottoposti all'attenzione della Cour de cassation) nel procedimento cagliaritano la moglie risultasse non residente nello Stato del foro.
} 
avevano fatto legittimo affidamento in particolar modo nel momento in cui avevano contratto nuove nozze (ciò, malgrado il pregiudizio eventualmente patito dalla donna in tale ipotesi, a onor del vero, non avrebbe comunque potuto rappresentare per lei un ostacolo reale, trattandosi di cittadina straniera residente all'estero). Nondimeno, la Corte cagliaritana opera una valutazione in cui il limite dell'ordine pubblico viene esaminato da un angolo visuale essenzialmente processuale, ed è in tale prospettiva che si evidenzia come la normativa egiziana applicata nel caso di specie, pur discriminando oggettivamente fra le parti in ragione del sesso quanto ai diritti che possono essere fatti valere ai sensi dell'istituto del talaq (basti pensare alla moglie è direttamente precluso di farvi ricorso) ${ }^{64}$, preveda che la pronuncia del ripudio debba essere portata a conoscenza della moglie affinché quest'ultima - pur non potendosi parlare di un vero e proprio contraddittorio, seppur differito, poiché la donna non ha alcuna possibilità di opporsi allo scioglimento del vincolo - possa attivarsi al fine di sollecitare un riconciliazione, oppure far valere in sede processuale le proprie ragioni di ordine economico.

35. Più che la mancata violazione del principio del contraddittorio e dei diritti essenziali della difesa, o del principio di eguaglianza tra i generi o, ancora, l'idea che la vigenza tra Egitto ed Italia della Convenzione dell'Aja del 1970 possa in qualche modo aver ristretto la portata dell'eccezione di ordine pubblico utilmente opponibile alla domanda di riconoscimento di un provvedimento straniero ${ }^{65}-$ come $^{2}$ pure sostiene il collegio cagliaritano -, la conclusione cui perviene la Corte d'appello pare sostanzialmente condivisibile proprio in considerazione delle specifiche circostanze del caso concreto, alla luce delle quali si è ragionevolmente ritenuto che il provvedimento straniero, e gli effetti che quest'ultimo, se riconosciuto, sarebbe stato in grado di esprimere nell'ordinamento italiano, non avrebbero potuto arrecare alcun pregiudizio alla situazione del soggetto alla cui tutela l'eventuale diniego avrebbe dovuto essere finalizzato ${ }^{6}$.

36. Tale pronuncia sembra dimostrare in modo inequivoco come, conformemente ai già menzionati principi generali desumibili dalla riforma del sistema italiano di diritto internazionale privato del 1995, la predisposizione di soluzioni precostituite e fondate su di una valutazione operata squisitamente in astratto - al pari di quanto fatto dalla Cassazione con la recente sentenza n. 16804 del 7 agosto 2020 ed il relativo principio di diritto in tema di ripudio - si riveli de iure non conforme al dettato normativo di riferimento, e de facto inopportuna poiché, escludendo un'attenta valutazione individualizzata, operata caso per caso in relazione alle specificità del rapporto dedotto in giudizio, potenzialmente pregiudizievole (o, comunque, incapace di garantire qualsiasi concreto vantaggio nei confronti) di quegli stessi interessi che si vorrebbero tutelare.

37. Rimandando al successivo paragrafo per una più estesa riflessione a quest'ultimo proposito, preme evidenziare come la stessa Corte di cassazione, con una pronuncia pressoché coeva e provenien-

${ }^{64}$ Non pare convincente, invece, l'osservazione di natura sostanziale relativa all'esistenza di un «uguale diritto (unilaterale)» della donna, che troverebbe espressione nel divorzio cd. khul: si tratta di una facoltà ben distinta rispetto a quella rimessa alla disponibilità del marito (basti pensare che, in tal caso, la donna rinuncia a qualsiasi pretesa di ordine economico: cfr. A. Mezghani, "Le juge français et les institutions du droit musulman", cit., p. 749). E, in ogni caso, non può ritenersi dirimente una considerazione legata unicamente alla formulazione in termini astratti del diritto straniero.

${ }^{65}$ Il riferimento è alla Convenzione dell'Aja del $1^{\circ}$ giugno 1970 sul riconoscimento dei divorzi e delle separazioni personali, la cui ratifica è stata autorizzata con 1.10 giugno 1985 n. 301, e che è in vigore per l'Italia a decorrere dal 20 aprile 1986 . Si veda, in particolare, l'art. 10 dello strumento, a tenor del quale «[c]iascuno Stato contraente può rifiutare il riconoscimento di un divorzio o di una separazione personale se è manifestamente incompatibile con il suo ordine pubblico» (nel testo della traduzione italiana comparsa in Gazz. Uff. n. 149 del 26 giugno 1985 - Suppl. ord.): in base ad esso, secondo la Corte d'appello, «il concetto di ordine pubblico deve intendersi ridotto al suo nucleo essenziale, cioè correlato a principi veramente irrinunciabili e fondamentali». Non solo non è del tutto chiaro in che modo il concetto di ordine pubblico così espresso si discosterebbe da quello di cui alle norme di diritto comune codificate nella 1. 218/1995, ma occorre altresì rilevare come la Convenzione dedichi altra ed apposita norma (l'art. 8) alla facoltà di diniego del riconoscimento fondata sulla violazione del diritto al contraddittorio e/o dei diritti essenziali della difesa, al pari di quanto prevede l'art. 65 della 1. 218/1995.

${ }^{66}$ A tal riguardo cfr. ancora le osservazioni svolte da G. PerLingIERI, G. ZARra, Ordine pubblico interno e internazionale tra caso concreto e sistema ordinamentale, cit., spec. pp. 152-153. 
te dalla medesima Prima sezione (seppur in differente composizione), paia aver svolto considerazioni difficilmente conciliabili con le motivazioni e con il principio di diritto espressi nella sentenza n. 16804 .

38. Il riferimento è all'ordinanza del 14 agosto $2020^{67}$ con la quale la Suprema Corte ha avuto modo di confrontarsi con un istituto di matrice confessionale islamica proprio dell'ordinamento giuridico iraniano, il divorzio cd. rojee, attivabile (unicamente) ad opera del marito ai sensi del codice civile iraniano. Il tema rileva in questa sede perché, nell'apprezzamento dei giudici di legittimità, si tratterebbe di una fattispecie che, «per il suo carattere unilaterale ed arbitrario, non si discosta dall'istituto del ripudio».

39. Va anzitutto premesso che, a quanto si apprende dall'ordinanza in questione, lo scioglimento del matrimonio sarebbe stato sancito per mezzo di un provvedimento proveniente dalla Corte Suprema di Teheran ed avente, quindi, indubbia natura giurisdizionale nell'ordinamento d'origine; allo stesso tempo, però, dalla sola lettura dell'ordinanza non è possibile inferire alcune informazioni che risulterebbero di rilievo ai fini di una più compiuta valutazione del giudizio espresso dalla Corte: è ad es. il caso della cittadinanza delle parti, o delle specifiche doglianze avanzate dalla donna e del relativo fondamento alla luce delle specificità del procedimento celebrato all'estero. Nondimeno, emerge con chiarezza come il giudizio italiano sia originato dal ricorso introdotto dalla moglie avverso l'avvenuta trascrizione del divorzio nei registri di stato civile. A seguito dell'accoglimento dell'istanza da parte della Corte d'Appello di Bari, e del conseguente ordine di cancellazione della trascrizione da essa impartito, il marito ha dunque proposto ricorso per Cassazione lamentando, in estrema sintesi, la violazione e falsa applicazione di legge in cui sarebbe incorso il collegio di merito in ragione $(i)$ del mancato scrutinio in ordine agli effetti della sentenza trascritta, nonché (ii) della scelta di motivare le proprie conclusioni su di un ragionamento che è parso fondarsi, al contrario, su di un apprezzamento tutto incentrato sulla (mera) lettera della pertinente normativa iraniana, trascurandone peraltro alcuni aspetti essenziali (ad es. in ordine all'interpretazione corrente della stessa, o al concreto svolgimento del giudizio nel caso di specie).

40. Ora, al di là di un lessico ed una sintassi a tratti ermetici, l'ordinanza in questione, nell'accogliere il ricorso e disporre il rinvio alla Corte d'appello pugliese in altra composizione, sviluppa taluni passaggi argomentativi che paiono assai lontani da quelli rinvenibili nella sentenza depositata appena sette giorni prima; passaggi che risulta dunque necessario richiamare nell'ottica della presente analisi. Preme in particolare evidenziare come, secondo il collegio, «quando il giudizio di delibazione [sic] si incentri sul requisito dell'ordine pubblico, la valutazione che si richiede al giudice deve mantenersi fedelmente aderente al dettato normativo dell'art. 64, comma 1, lett. g), 1. 218/1995, secondo cui il riconoscimento non può avere luogo se le sue disposizioni producono "effetti contrari all'ordine pubblico" e, di conseguenza, occorre che il giudice, senza estendere la propria cognizione aliunde, valuti "gli 'effetti' della decisione nel nostro ordinamento e non la correttezza della soluzione adottata alla luce dell'ordinamento straniero o della legge italiana, non essendo consentita un'indagine sul merito del rapporto giuridico dedotto" (Cass., Sez. I, 18/04/2013, n. 9483) ${ }^{68}$. Dunque, nel ribadire il pacifico divieto di (esame e) riesame nel merito che connota in generale il procedimento innanzi alla Suprema Corte e, in particolare, quello sul riconoscimento di una pronuncia straniera, la Cassazione pare altresì ritornare ad una posizione più aderente e fedele al dettato della 1. 218/1995 di riforma del sistema italiano di diritto internazionale privato. E che tale sia proprio l'idea da cui muove la sentenza del 14 agosto 2020 emerge, ancor più nitidamente, dal richiamo a quei precedenti in occasione dei quali la stessa Corte aveva evidenziato come il fatto che una pronuncia straniera applichi una disciplina materiale conforme o difforme a norme interne, «benché imperative o inderogabili» ${ }^{69}$, non può di per sé rappresentare un

\footnotetext{
${ }^{67} \mathrm{Si}$ tratta di Cass. civ., I sez., ordinanza 14 agosto 2020, n. 17170, cit.

${ }^{68}$ Punto 7 della motivazione. Enfasi aggiunta.

${ }^{69} \mathrm{Si}$ pensi al caso della norma di applicazione necessaria relativa al requisito dell'età per il matrimonio dei cittadini italiani (derivante dal combinato disposto degli artt. 115 e 84 c.c.): in materia, sia consentito il rinvio a F. Pesce, "I child marriages tra tutela dei diritti e portabilità degli status", Diritti umani e diritto internazionale, 2021, in corso di pubblicazione.
} 
ostacolo sufficiente a determinare il rigetto del riconoscimento ${ }^{70}$, posto che, diversamente opinando, «le norme di conflitto ${ }^{71}$ sarebbero operanti solo ove conducessero all'applicazione di norme materiali aventi contenuto simile a quelle italiane, (...) rendendo inutili le norme di diritto internazionale privato ${ }^{72}$. Coerentemente con tali premesse - ma allo stesso tempo arrivando sostanzialmente a smentire se stessa, rispetto a quanto affermato nell'ambito della sentenza depositata sette giorni prima -, la Prima Sezione critica la Corte d'appello di Bari per non aver «fatto mistero di ritenere che, per il fatto, in particolare, di riconoscere una posizione di privilegio del marito rispetto alla moglie, (...) il divorzio iraniano realizzi un modello giuridico inconciliabile con le "regole inderogabili e fondamentali immanenti ai più importanti istituti giuridici nazionali"», sicché «[c]osì ragionando non solo mostra di far propria una convinzione in materia di ordine pubblico che non trova più riscontro nel diritto vivente, ma quel che più conta mostra di coltivare un'esegesi del limite concretamente operante nel giudizio di delibazione [sic] che non era praticabile (...) neppure prima che la nozione di ordine pubblico fosse oggetto dell'evoluzione in senso estensivo di cui s'è dato sopra conto» ${ }^{73}$.

41. Come accennato, permangono alcuni aspetti dubbi, anche in punto di diritto, rispetto ai quali la sola lettura dell'ordinanza non risulta dirimente (non è chiaro, ad esempio, se anche nel giudizio di merito sia stato $a b$ origine escluso qualsiasi riferimento all'art. 65 della 1. 218/1995, oltre all'art. 64; né se la moglie abbia altresì lamentato la violazione delle lett. $b$ e/o $c$ del medesimo art. 64, in aggiunta alla violazione dell'ordine pubblico ex lett. $g$ ). Ciononostante, e limitatamente a quanto si è evidenziato poc'anzi, la decisione rappresenta senz'altro un modello alternativo rispetto a quello della precedente sentenza n. 16804; modello, dunque, da tener in attenta considerazione onde verificare quali dei due percorsi argomentativi sarà destinato a trovare maggior successo nell'ambito della giurisprudenza a venire.

\section{Quali prospettive per il riconoscimento dei ripudi stranieri? Per un "temperamento" della chiusura in chiave multiculturalista}

42. Quanto sin qui osservato circa allo stato dell'arte in tema di riconoscimento dei provvedimenti stranieri di ripudio consente di trarre alcune considerazioni conclusive, anche in chiave prospettica: in un contesto storico - qual è quello attuale - caratterizzato come noto da un costante aumento della mobilità internazionale delle persone, il tema della "portabilità" transfrontaliera degli status familiari ${ }^{74}$ assume via via un interesse teorico ed un rilievo pratico sempre maggiori.

43. A tal riguardo non può non rilevarsi come, aderendo alla posizione fatta propria dalla Cassazione con la sentenza n. 16804 del 7 agosto 2020 e fondata su di un apprezzamento di tipo astratto, inerente al contenuto sostanziale della norma straniera applicata ed alla posizione processuale rivestita dalle parti, non potrebbe che sostenersi l'idea di un'incompatibilità ontologica del ripudio con il nostro ordinamento giuridico; tuttavia, come si è cercato di evidenziare, così facendo si andrebbe ad escludere quell'apprezzamento, richiesto in termini inequivoci dalla vigente disciplina internazionalprivatistica di diritto comune ${ }^{75}$, che attiene agli effetti concreti che il provvedimento straniero, se riconosciuto, sarebbe in grado di esprimere nel nostro ordinamento, e che implica necessariamente un esame individualizzato di ciascuna istanza.

\footnotetext{
${ }^{70}$ In questi termini si era espressa Cass., 30 settembre 2016, n. 19599, cit.

${ }^{71}$ Il riferimento alle sole norme di conflitto non pare, in realtà, del tutto appropriato, posto che si sta trattando di previsioni di diritto processuale civile internazionale relative al riconoscimento delle sentenze straniere, e che il riconoscimento si realizza attraverso la tecnica conflittuale solo nella limitata ipotesi di cui all'art. 65 della 1. 218/1995.

${ }^{72}$ Cass., 4 maggio 2007, n. 10215, in Rivista di diritto internazionale privato e processuale, 2008, pp. 214 ss.

${ }^{73}$ Punto 9 della motivazione. Enfasi aggiunte.

${ }^{74}$ Con particolare riguardo alla dimensione intraeuropea del fenomeno, cfr. S. PFEIFF, La portabilité du statut personnel dans l'espace européen, Bruxelles, Bruylant, 2017.

${ }^{75}$ Cfr. 1. 218/1995, art. 64, lett. $g$.
} 
44. In altri termini, la pronuncia sembrerebbe legittimare l'idea di una risposta negativa da opporsi de plano ad ogni eventuale domanda di riconoscimento di un ripudio, evitando persino di coinvolgere l'autorità giurisdizionale preposta ad operare il vaglio sulla riconoscibilità di sentenze e provvedimenti stranieri. Vero è che il panorama offerto dagli ordinamenti giuridici di stampo confessionale islamico è assai variegato quanto ai rispettivi poteri riconosciuti ai due coniugi ed al ruolo della moglie nell'ambito del procedimento ma, nondimeno, rimane ferma la discriminazione tra le posizioni delle due parti che l'istituto del ripudio opera per sua stesa natura: rispetto ad essa, anche un'eventuale approvazione ex post da parte della donna, seppur genuinamente prestata, non pare sufficiente a garantire un riequilibrio tale da poter qualificare l'istituto in questione come astrattamente conforme ai principi fondamentali dell'ordinamento interno.

45. Ne discende che, a fronte di una valutazione in ordine alla mera conciliabilità tra i principi giuridici cui risulta ispirato il diritto materiale di famiglia degli ordinamenti interessati, da un lato, e dello Stato italiano, dall'altro lato, la conclusione cui approda la sentenza n. 16804 parrebbe necessitata.

46. E non solo. Se davvero si vuole accedere al postulato dell'assoluta irriconoscibilità del ripudio, ipotizzato dalla pronuncia, occorre altresì considerare che l'istanza di riconoscimento non può che essere rigettata - sempre - ancor prima di pervenire in sede giurisdizionale: è infatti l'ufficiale di stato civile a poter (e dover) negare la trascrizione in prima battuta, ogniqualvolta abbia ragione di ipotizzare l'esistenza di un motivo ostativo rispetto ad essa, ivi incluso il contrasto con l'ordine pubblico ${ }^{76}$. E sempre partendo da tali premesse l'eventuale e successiva opposizione a tale rifiuto, da proporsi innanzi alla Corte d'appello competente per territorio conformemente a quanto previsto in via generale dall'artt. 67 della 1. 218/1995, dovrebbe peraltro ritenersi, inevitabilmente, destinata a condurre ad un esito parimenti negativo.

47. Ora, fermo restando che un'azione giudiziaria di tal sorta non avrebbe ragion d'essere e rappresenterebbe un aggravio in termini economici a fronte del quale non sussisterebbe una reale possibilità di accoglimento, vale la pena evidenziare che l'orientamento giurisprudenziale qui considerato consegnerebbe all'ufficiale di stato civile una direttiva chiara ed inequivoca nel senso del diniego della trascrizione. E se è vero che dal Massimario per l'ufficiale di stato civile, predisposto dal Ministero dell'Interno, già emerge nitidamente proprio tale linea guida ${ }^{77}$, si avrà modo di evidenziare come sussistano talune ipotesi concrete a fronte delle quali una valutazione più attenta, ed individualizzata, risulterebbe quanto mai opportuna già in prima battuta, ossia proprio all'interno dell'ufficio proposto alla conservazione dei registri di stato.

48. La valutazione circa gli effetti della sentenza straniera che l'art. 64, lett. $g$, della 1. 218/1995 richiede al fine di poter attivare il filtro dell'ordine pubblico, dovrebbe infatti fungere da criterio-guida per tutti coloro che siano chiamati a garantire il riconoscimento della pronuncia in questione, ivi inclusi gli ufficiali di stato civile, laddove si tratti di provvedere alla sua attuazione per il tramite della trascrizione nei pubblici registri. Il divieto di trascrizione, impartito agli ufficiali di stato nell'ambito del Massimario, determina al contrario l'obbligo di avviare in ogni caso un procedimento in sede giurisdizionale; e, come già si è avuto modo di evidenziare, muovendo da simili premesse risulta poi difficile comprendere in che modo la competente Corte d'appello potrebbe eventualmente concludere per il riconoscimento di una decisione straniera fondata su di un istituto «che risulta in contrasto con il nostro ordinamento e con principi inderogabili di ordine pubblico» ${ }^{78}$.

\footnotetext{
${ }^{76}$ Valgono infatti, anche in questa sede, i motivi ostativi al riconoscimento delle sentenze e dei provvedimenti stranieri previsti dagli artt. 64 e 65 della 1. 218/1995. È l'ipotesi che si è verificata, ad es., nel caso su cui si è pronunciata la Corte d'appello di Cagliari nel 2008 (cfr. par. precedente).

${ }^{77}$ Cfr. Massimario, cit., sub 11.3.1. (pag. 148)

${ }^{78}$ Ibidem.
} 
49. Ebbene, si ritiene che un quadro di tal sorta sia da considerarsi iniquo e non rispondente ad alcun interesse meritevole di tutela: né a quello dell'ordinamento richiesto, nel suo insieme (nella specie, quello italiano), né a quello dei soggetti direttamente coinvolti, ivi inclusa la parte "debole" che attraverso tale reazione di chiusura si intenderebbe proteggere, ossia la donna. È però necessaria una precisazione: tale affermazione è da riferirsi solo ed esclusivamente all'ipotesi di una chiusura generalizzata ed aprioristica dell'ordinamento rispetto ad ogni possibile istanza attraverso la quale venga richiesto l'attribuzione di effetti, in Italia, ad un provvedimento straniero con cui si sia sancito lo scioglimento di un vincolo coniugale per ripudio.

50. In altri termini, innanzi a (e sempre purché si tratti di) uno stato libero legittimamente acquisito all'estero e, dunque, già consolidatosi all'interno di un altro ordinamento ${ }^{79}$, si intende figurare l'esistenza di particolari circostanze al ricorrere delle quali non vi sarebbe alcuna valida ragione per escludersi il riconoscimento, atteso che o non ne deriverebbe alcun pregiudizio per la donna oppure, addirittura, l'unico pregiudizio figurabile per la posizione di quest'ultima discenderebbe proprio dal diniego del riconoscimento.

51. Rientrano nella prima ipotesi, ad esempio, quei casi in cui la donna, avente unicamente la cittadinanza straniera, a seguito del ripudio (incontestato) e dopo aver percepito il conseguente indennizzo economico, abbia mantenuto la propria residenza nel Paese d'origine e qui abbia seguitato a condurre la propria vita quale soggetto di stato libero, e senza dar luogo ad alcun tipo di contatto con l'ordinamento italiano. Qualora l'ex marito abbia invece trasferito in Italia la propria residenza e, successivamente, creato qui un nuovo nucleo familiare, si può presumere che dal riconoscimento del ripudio non deriverebbe alcun pregiudizio a carico dell'ex moglie

52. La seconda ipotesi, assai più delicata, si può invece ben comprendere pensando al caso della donna ripudiata che abbia trasferito la residenza nel nostro Paese e qui desideri iniziare una nuova vita familiare: il diniego del riconoscimento innanzi ad un'istanza proveniente dalla stessa donna ripudiata pare essere un esito "estremo" che risulta difficile da giustificare ${ }^{80}$. Stessa situazione - ed anzi ancor più grave - può verificarsi nel caso di un rapporto matrimoniale all'interno del quale la donna ha patito violenze di natura psicologica e/o fisica e del quale, dunque, ha tutto l'interesse di riconoscersi liberata, senza inutili appesantimenti procedimentali.

53. È altresì evidente che analoghe considerazioni non valgono a fronte di tutti quei casi in cui la moglie si sia opposta al ripudio o, comunque, intenda opporsi al riconoscimento dello stesso. Ma proprio per tale ragione vale forse la pena di considerare i benefici che potrebbero derivare da un approccio più flessibile e ragionato alla materia in questione, fermo restando il mantenimento di un altro grado di tutela per i diritti fondamentali del soggetto meritevole di protezione, poiché potenzialmente pregiudicato, ossia la donna: si tratterebbe di mitigare la reazione dell'ordinamento, ammettendo il riconoscimento degli effetti del ripudio, in quei soli casi in cui ciò non risulti contrario agli interessi di quest'ultima, o si riveli addirittura rispondente ad essi.

54. Una soluzione di questo tipo non sembra contrastare con le previsioni della 1. 218/1995 dedicate al riconoscimento delle sentenze straniere. Considerando le due censure che vengono mosse avverso le istanze di riconoscimento di un ripudio, cioè il contrasto con l'ordine pubblico, da un lato, e la violazione dei diritti fondamentali della difesa e dell'integrità del contraddittorio tra le parti, dall'altro, occorre osservare quanto segue.

\footnotetext{
${ }^{79}$ Cfr. C. CAmpiglio, "Identità culturale, diritti umani e diritto internazionale privato", cit., p. 271.

${ }^{80} \mathrm{Si}$ tratta, ad es., della situazione esaminata dal Tribunale di Tunisi con sentenza del 27 giugno 2000 e ricordata da L. Gannagé, "Le relativisme des droits de l'homme dans l'espace méditerranéen", Revue international de droit comparé, 2006, spec. pp. 114 ss.: nel caso di specie, la richiesta di riconoscimento era stata rigettata.
} 
55. Sotto il primo profilo, infatti, si è già evidenziato in più di un'occasione come la riforma del sistema italiano di diritto internazionale privato del 1995 abbia esplicitato la necessità di condurre un'accurata valutazione circa gli effetti che il provvedimento straniero è passibile di produrre nel contesto dell'ordinamento interno. Ciò - lo si è visto - fa sì che non sia ab origine precluso il riconoscimento di una sentenza con cui si dà applicazione ad istituti giuridici che, pure, siano fondati su principi incompatibili con quelli posti alla base dei corrispondenti istituti del diritto materiale italiano. E non solo. Occorre altresì notare che, laddove scelga di aderire all'idea di quella particolare connotazione che il limite in parola assume laddove operi quale ordine pubblico cd. attenuato ${ }^{81}$, se ne possono trarre argomenti a favore di un arretramento dello stesso, innanzi a status e rapporti legittimamente già insorti e consolidatosi nell'ambito di un ordinamento straniero; ordinamento con cui i soggetti coinvolti presentano significativi elementi di contatto ${ }^{82}$.

56. Non sembra convincente neppure la tesi - che, pure, ciclicamente viene ripresa in giurisprudenza, e che viene prospettata inter alia anche dalla sentenza n. 16804 - secondo cui uno specifico ed autonomo profilo di frizione con l'ordine pubblico sarebbe rappresentato dal mancato accertamento, da parte dell'autorità giurisdizionale $a$ quo, in ordine all' 'effettiva cessazione del rapporto affettivo e di convivenza dei coniugi ovvero della possibilità di una sua composizione o continuazione» ${ }^{83}$. Tali considerazioni, pur identificando un principio che parrebbe essere stato legittimamente ascrivibile alla nozione di ordine pubblico per come rinvenibile in un differente momento storico, si ritengono non più attuali: anche a voler tralasciare le recenti, e rilevanti, riforme intervenute nel diritto materiale di famiglia italiano - che pure sembrerebbero proprio dar conto di un'evoluzione in senso privatistico e, dunque, consensualistico dell'istituto del divorzio ${ }^{84}$-, vale la pena di ricordare come esistano ordinamenti appartenenti al cd. spazio giudiziario europeo nell'ambito dei quali lo scioglimento del vincolo matrimoniale può riposare sulla mera volontà espressa dalle parti, senza alcuna necessità di indagine in ordine al venir meno della "comunione materiale e spirituale" fra i coniugi o alla possibilità di una composizione della crisi ${ }^{85}$. Ebbene, se veramente quello poc'anzi richiamato corrispondesse ad un principio di ordine pubblico internazionale del nostro Paese, esso non potrebbe che essere fatto valere anche nei confronti di decisioni provenienti da Stati membri dell'Unione europea, atteso che il regolamento 2201/2003 (cd. Bruxelles IIbis), sub art. 22, lett. $a^{86}$, introduce proprio l'ipotesi della manifesta contra-

${ }^{81}$ In argomento cfr. D. SINDRES, "Vers la disparition de l'ordre public de proximité?”, Clunet, 2012, pp. 887 ss.; O. FERACI, L'ordine pubblico nel diritto dell'Unione europea, Milano, Giuffrè, 2012, pp. 14 ss.

${ }^{82}$ Tanto è vero che, proprio con riguardo alla teoria dell'effetto attenuato dell'ordine pubblico, nel 2005 l'Institut de droit international ha adottato una nota risoluzione per mezzo della quale ha espresso l'avviso che il diniego del riconoscimento di un ripudio possa aver luogo solo nel caso in cui la moglie sia (o sia stata) cittadina dello Stato in cui il riconoscimento è richiesto, o vi sia abitualmente residente al momento dell'istanza. E non solo. Il diniego non potrebbe comunque intervenire qualora la donna abbia acconsentito al ripudio, o sia stata adeguatamente indennizzata in termini pecuniari. V. Résolution - Neuvième Commission - Différences culturelles et ordre public en droit international privé de la famille, 25 agosto 2005, Rapporteur P. Lagarde, https://www.idi-iil.org/app/uploads/2017/06/2005_kra_02_fr.pdf.

${ }^{83}$ Cfr. par. 2.8. della sentenza in parola.

${ }^{84}$ Quanto meno in assenza di figli: si fa riferimento alle nuove possibilità offerte dalla negoziazione assistita e dall'accordo concluso innanzi all'ufficiale di stato civile. Come già si è evidenziato, a tal riguardo l'accordo non è però unanime: cfr. ad es. P. Virgadamo, "Ripudio islamico e contrarietà all'ordine pubblico tra unitarietà del limite e corretta individuazione dei principi”, cit., p. 362, nota 43. V'è chi ritiene, al contrario, che tale risultato si fosse già raggiunto con l'introduzione, nel nostro ordinamento, del divorzio su richiesta congiunta dei coniugi: cfr. G. PerlingIERI, G. ZARRA, Ordine pubblico interno e internazionale tra caso concreto e sistema ordinamentale, cit., p. 151; P. PICONE, "L'art. 65 della legge italiana di riforma del diritto internazionale privato e il riconoscimento delle sentenze straniere di divorzio", Rivista di diritto internazionale privato e processuale, 2000 , p. 393.

${ }^{85} \mathrm{Ne}$ è un perfetto esempio l'ordinamento svedese (a tal riguardo si vedano le informazioni messe a disposizione dalla Rete giudiziaria europea in materia civile e commerciale alla pagina https://beta.e-justice.europa.eu/45/IT/divorce_and_legal_separation?SWEDEN\&member=1).

${ }^{86} \mathrm{Ma}$ lo stesso discorso vale con riguardo al più recente strumento normativo che è destinato a sostituirlo, ossia il regolamento (UE) 2019/1111 del Consiglio, del 25 giugno 2019, relativo alla competenza, al riconoscimento e all'esecuzione delle decisioni in materia matrimoniale e in materia di responsabilità genitoriale, e alla sottrazione internazionale di minori, GUUE L 178 del 2 luglio 2019, p. 1 (al cui interno l'ordine pubblico, quale limite al riconoscimento di decisioni in materia di scioglimento del vincolo matrimoniale, viene ripreso dall'art. 38 , lett. $a$ ). 
rietà con l'ordine pubblico dello Stato richiesto quale possibile limite al riconoscimento di pronunce rese in altri Paesi membri. E se da un lato non risulta che tale eventualità si sia mai verificata e che, per altro verso, una deroga di tal natura alla libera circolazione intraeuropea delle decisioni potrebbe essere ritenuta conforme con il diritto dell'UE da parte della Corte di giustizia, essa sembra altresì essere stata esclusa in dottrina ${ }^{87}$. Ulteriori conferme a tale conclusione possono essere tratte anzitutto dal già citato Massimario per l'ufficiale di stato civile, nel contesto del quale si evidenzia come le sentenze di separazione o divorzio ricadenti nell'ambito applicativo del regolamento Bruxelles IIbis possano essere trascritte in Italia senza che sia necessario produrne copia conforme, essendo al contrario sufficiente la produzione del certificato di cui all'art. 39 del regolamento, compilato sulla base del modello standard di cui all'allegato $\mathrm{I}^{88}$ : ne discende che è materialmente impossibile, in tal caso, operare qualsiasi tipo di controllo in ordine alle motivazioni su cui la decisione straniera riposa. Milita inoltre in tal senso quella giurisprudenza di merito con cui è stata data diretta applicazione alle norme marocchine ed albanesi che contemplano il divorzio immediato per maltrattamenti ${ }^{89}$.

57. Quanto, poi, al secondo dei profili accennati, ossia quello relativo alla violazione dei diritti fondamentali della difesa e dell'integrità del contraddittorio tra le parti, va osservato che, quand'anche tali diritti appaiano concretamente pregiudicati nel corso del procedimento celebrato nell'ordinamento $a$ quo (e tale potrebbe essere stato il caso di cui alla sentenza n. 16804, malgrado essa, così come il principio di diritto enunciato in tale occasione, non paiono diffondersi in merito a questo particolare aspetto, che pure risulta valutato in via autonoma ad opera della Corte d'appello) ${ }^{90}$, considerazioni di ordine teleologico e sistematico sembrerebbero precludere la possibilità che la tutela di un diritto sia perseguita in modo così avulso dalla realtà concreta della singola fattispecie, da poter arrivare al punto di ritorcersi contro lo stesso soggetto che si intenderebbe tutelare, pregiudicandone la posizione. Si tratta di un principio che dovrebbe egualmente ispirare non soltanto l'applicazione del limite dell'ordine pubblico (peraltro eventualmente nella sua connotazione "attenuata" di cui s'è detto poc'anzi), ma anche quella dei motivi ostativi più specificamente inerenti allo svolgimento del procedimento nello Stato d'origine, ossia proprio quelli relativi al rispetto dei diritti all'integrità del contraddittorio e di difesa. Neanche sotto quest'ultimo angolo visuale, infatti, dovrebbero realizzarsi automatismi tali da determinare il rigetto dell'istanza di riconoscimento per il solo fatto che il soggetto controinteressato non è stato messo nelle condizioni di esercitare appieno, ed utilmente, il diritto di resistere alle pretese di chi ha originariamente introdotto il giudizio. E se nel caso del ripudio palestinese, esaminato dalla Suprema Corte, simili considerazioni non sembrano poter condurre a conclusioni diverse da quella del rigetto del ricorso, e dunque del riconoscimento, fatta propria dal collegio (atteso che la donna si è inequivocabilmente opposta alla trascrizione, peraltro avanzando circostanziate censure proprio in ordine alla lesione dei propri diritti di difesa), si possono dare casi rispetto ai quali l'interesse - in concreto - della moglie ripudiata sarebbe rappresentato proprio e solo dal riconoscimento dell'atto con cui l'unione coniugale è stata sciolta nel Paese d'origine, pur senza la sua partecipazione attiva. Sulla scorta di tali considerazioni, si ritiene dunque di evidenziare l'irragionevolezza degli esiti che possono derivare dal condurre alle estreme conseguenze le tesi sposate dalla Cassazione nella sentenza n. 16804 e compendiate dal principio di diritto formulato in quella sede: ne discenderebbe infatti l'obbligo, per il giudice italiano, di negare il riconoscimento del ripudio perfino innanzi a fattispecie in cui la donna

\footnotetext{
${ }^{87}$ Cfr. K. Siehr, “Article 22”, in U. Magnus, P. Mankowski (eds.), European Commentaries on Private International Law (ECPIL). Volume IV - Brussels IIbis Regulation, Köln, Otto Schmidt, 2017, p. 294.

${ }^{88}$ Massimario per l'ufficiale di stato civile (ediz. 2012), sub 11.2.

${ }^{89} \mathrm{Si}$ tratta infatti di scioglimenti immediati ed invocati su base unilaterale: cfr. Trib. Pordenone, 14 settembre 2005, in Rivista di diritto internazionale privato e processuale, 2006, p. 181; Trib. Tivoli, 14 novembre 2002, ivi, 2003, p. 534.

${ }_{90}$ Tuttavia non pare affatto convincente, a tal riguardo, la tesi sostenuta dal ricorrente, secondo cui la mera comparizione della moglie innanzi al tribunale sciaraitico farebbe venir meno la violazione dei diritti essenziali della difesa di cui all'art. 64, lett. $b$ della 1. 218/1995. Emerge infatti dagli atti che la donna era comparsa innanzi all'organo giurisdizionale al solo fine di vedersi formalmente notificato il provvedimento di ripudio e, soprattutto, senza godere di alcun potere di opposizione al ripudio in modo utile, ossia con una reale possibilità di ottenerne la riconsiderazione.
} 
abbia prestato il proprio consens $0^{91} \mathrm{o}$, addirittura, abbia essa stessa sollecitato il ripudio e/o richiesto il suo riconoscimento ${ }^{92}$.

58. È appena il caso di ricordare come le osservazioni fin qui svolte con riferimento ai motivi ostativi di cui alle lett. $b, c$ e $g$ dell'art. 64 della 1. 218/1995, possono essere egualmente trasposte, mutatis mutandis, al caso in cui si discuta dell'applicazione del successivo art. 65 e del relativo riconoscimento "semplificato" per il tramite delle norme di conflitto, posto che tali limiti all'ingresso dei provvedimenti stranieri rappresentano in realtà il nucleo comune ad entrambe le previsioni ${ }^{93}$.

59. Preme conclusivamente ribadire, con riguardo alla sentenza della Suprema Corte n. 16804 del 7 agosto 2020, che se da un lato non si ravvisano solidi argomenti per sostenere che, nel caso di specie, si sarebbe dovuto garantire il riconoscimento del talaq palestinese, dall'altro lato il percorso argomentativo che conduce a tal esito risulta non soltanto opinabile ma - in ragione della scelta di enucleare un principio di diritto a sua volta non esente da critiche - anche foriero di orientare in un senso eccessivamente rigido ed "aprioristicamente garantista" la futura giurisprudenza relativa agli effetti dei ripudi stranieri.

60. La posizione più ragionevole ed attenta ai (reali) interessi dei soggetti coinvolti appare, al contrario, assai prossima a quella curiosamente indicata dalla stessa Sezione Prima della Corte di cassazione, con l'ordinanza depositata a soli sette giorni di distanza da quella sentenza ${ }^{94}$ : la reazione dell'ordinamento interno innanzi all'istanza di riconoscimento di un provvedimento straniero, pure ispirato a principi ed adottato in forza di previsioni non compatibili con quelli del foro, non dovrebbe sostanziarsi in una chiusura aprioristica a pregiudiziale. Al contrario, la tutela dei diritti meritevoli di protezione dovrebbe calarsi nella realtà concreta e determinare l'arretramento dei motivi ostativi al riconoscimento a fronte (e solo a fronte) di quelle situazioni in cui il tentativo di tutela, rimanendo eccessivamente ancorato al piano astratto, finirebbe per trasformarsi in un concreto pregiudizio a danno di quegli interessi che si vorrebbero protetti e garantiti ${ }^{95}$.

${ }^{91}$ Ed è proprio ciò che è avvenuto nel caso deciso con sentenza di App. Milano, 17 dicembre 1991, in Rivista di diritto internazionale privato e processuale, 1993, p. 109. A tal riguardo vale la pena di ricordare la soluzione adottata dal legislatore belga nel contesto del codice di diritto internazionale privato del 2004 (legge 16 luglio 2004, riprodotta in Rivista di diritto internazionale privato e processuale, 2005, pp. $231 \mathrm{ss}$.): in base all'art. 57 di quest'ultimo, infatti, gli atti di ripudio pronunciati all'estero possono essere eccezionalmente riconosciuti qualora siano stati omologati in sede giurisdizionale nell'ordinamento a quo e, al tempo stesso, risultino "deconnessi" (per cittadinanza o residenza dei coniugi) da ordinamenti in cui tali atti sono vietati, e purché la moglie acconsenta a tale riconoscimento in modo certo e senza costrizioni. A tal riguardo, malgrado R. CLERICI, "La compatibilità del diritto di famiglia mussulmano con l'ordine pubblico internazionale", cit., p. 203, ritenga che non sia affatto agevole giungere ad accertare una inequivocabile volontà della donna in tal senso, occorre pur considerare che vi sono ipotesi - come quella della richiesta espressa di riconoscimento da parte della donna stessa - in cui ciò è senz'altro possibile. Secondo C. CAMPIGLIO, "Identità culturale, diritti umani e diritto internazionale privato", cit., p. 273, le condizioni poste dal legislatore belga finiscono per rendere concretamente improbabile l'ipotesi del riconoscimento.

${ }_{92}$ A tal proposito C. CAMPIGLIO, "Identità culturale, diritti umani e diritto internazionale privato", cit., p. 273, ricorda la condivisibile posizione espressa dal Tribunale supremo spagnolo con pronuncia del 21 aprile 1998: in quella sede l'organo giurisdizionale aveva ritenuto che ragioni di giustizia sostanziale imponessero il riconoscimento, richiesto dalla donna, di un ripudio egiziano: «[m]antener lo contrario significaría elevar el formalismo del principio igualatorio por encima del resultado material que se produce en el caso concreto, convirtiendo en perjuicio lo que debiera actuar en protección de la mujer discriminada».

${ }^{93}$ Cfr. O. VAnin, "Ripudio islamico, principio del contraddittorio e ordine pubblico italiano", cit., spec. p. 1032.

${ }^{94}$ Cass. civ., I sez., ordinanza 14 agosto 2020, n. 17170, cit.

${ }^{95}$ Solo così potrebbe ulteriormente ritenersi garantito il rispetto di quel diritto alla vita privata e familiare che, accolto all'interno dell'art. 8 CEDU, ammette eventuali limitazioni ad opera degli Stati solo a valle di un rigoroso giudizio di bilanciamento: l'innalzamento del limite dell'ordine pubblico dovrebbe essere finalizzato al perseguimento di un interesse collettivo meritevole di tutela e, allo stesso tempo, non implicare un sacrificio intollerabile a carico delle posizioni soggettive individuali. 